\title{
Olive phenolic compounds: metabolic and transcriptional profiling during fruit development
}

\author{
Fiammetta Alagna ${ }^{1 *}$, Roberto Mariotti ${ }^{1}$, Francesco Panara ${ }^{1}$, Silvia Caporali ${ }^{2}$, Stefania Urbani ${ }^{2}$, Gianluca Veneziani ${ }^{2}$, \\ Sonia Esposto ${ }^{2}$, Agnese Taticchi ${ }^{2}$, Adolfo Rosati ${ }^{3}$, Rosa Rao ${ }^{4}$, Gaetano Perrotta ${ }^{5}$, Maurizio Servili ${ }^{2}$ \\ and Luciana Baldoni $i^{*}$
}

\begin{abstract}
Background: Olive (Olea europaea L.) fruits contain numerous secondary metabolites, primarily phenolics, terpenes and sterols, some of which are particularly interesting for their nutraceutical properties. This study will attempt to provide further insight into the profile of olive phenolic compounds during fruit development and to identify the major genetic determinants of phenolic metabolism.

Results: The concentration of the major phenolic compounds, such as oleuropein, demethyloleuropein, 3-4 DHPEA-EDA, ligstroside, tyrosol, hydroxytyrosol, verbascoside and lignans, were measured in the developing fruits of 12 olive cultivars. The content of these compounds varied significantly among the cultivars and decreased during fruit development and maturation, with some compounds showing specificity for certain cultivars. Thirty-five olive transcripts homologous to genes involved in the pathways of the main secondary metabolites were identified from the massive sequencing data of the olive fruit transcriptome or from CDNA-AFLP analysis. Their mRNA levels were determined using RT-qPCR analysis on fruits of high- and low-phenolic varieties (Coratina and Dolce d'Andria, respectively) during three different fruit developmental stages. A strong correlation was observed between phenolic compound concentrations and transcripts putatively involved in their biosynthesis, suggesting a transcriptional regulation of the corresponding pathways. OeDXS, OeGES, OeGE1OH and OeADH, encoding putative 1-deoxy-D-xylulose-5-P synthase, geraniol synthase, geraniol 10-hydroxylase and arogenate dehydrogenase, respectively, were almost exclusively present at 45 days after flowering (DAF), suggesting that these compounds might play a key role in regulating secoiridoid accumulation during fruit development.
\end{abstract}

Conclusions: Metabolic and transcriptional profiling led to the identification of some major players putatively involved in biosynthesis of secondary compounds in the olive tree. Our data represent the first step towards the functional characterisation of important genes for the determination of olive fruit quality.

Keywords: Olea europaea, Phenolics, Secoiridoids, RT-qPCR, Transcriptome, Secondary metabolism

\section{Background}

The olive fruit mesocarp accumulates a wide range of secondary metabolites. The main category of secondary metabolites is represented by secoiridoids, a group of monoterpenoids with a cleaved methylcyclopentane skeleton, which are typical of the Oleaceae and other few dicotyledonous families. Secoiridoids are abundant in olives as phenol-conjugated compounds that might contain a glycoside moiety. The most important secoiridoids

\footnotetext{
* Correspondence: fiammetta.alagna@igv.cnr.it; luciana.baldoni@igv.cnr.it 'CNR - Institute of Plant Genetics, 06128 Perugia, Italy

Full list of author information is available at the end of the article
}

of the olive fruit and virgin olive oil are oleuropein, demethyloleuropein, oleuroside, ligstroside, nüzhenide and their aglycon forms, such as the dialdehydic form of decarboxymethyl elenolic acid linked to either 3,4-DHPEA or p-HPEA (3,4-DHPEA-EDA and p-HPEA-EDA, respectively), an isomer of oleuropein aglycon (3,4-DHPEA-EA) and the ligstroside aglycon (p-HPEA-EA) [1]. The secoiridoid compound p-HPEA-EDA, also called oleocanthal, was identified in the extra-virgin olive oil but has never been observed in the fruits, probably as a consequence of post-harvest enzymatic activity [2]. Other olive phenolics include phenolic acids, phenolic alcohols (e.g.,

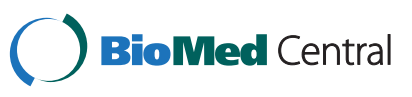


hydroxytyrosol (3,4-DHPEA) and tyrosol (p-HPEA), flavonoids and lignans [3]. These compounds are observed in all parts of the drupe, with the highest concentration in the pulp [4]. The fruits of several olive cultivars also contain high amounts of verbascoside [5] and other phenolics present at maturity, such as homovanillic alcohol, 3,4-dihydroxyphenylacetic acid (DHPAC), caffeic acid, p-coumaric acid, phloretic acid, vanillic acid [6] and low amounts of comselogoside [7].

Studies of the phenolic profiles in the mesocarp, exocarp, seed, stone and leaf of the olive have demonstrated that different tissues contain distinct compounds. For example, nüzhenide and salidroside are only observed in the olive seed [8], while the flavonoids luteolin-7glucoside, rutin and quercetin are exclusively present in the fruit peel [9].

Other compounds in the olive fruit include triterpenic acids such as maslinic and oleanolic acids [10] and tocopherols [11].

Squalene, an intermediate of the sterol pathway, is the precursor of $\alpha$ - and $\beta$-amyrins and the triterpenic diols erythrodiol and uvaol. Squalene is another important compound with recognised effects on human health that is present in consistent amount only in olive and other vegetable oils [12]. Sterols, such as $\beta$-sitosterol, cycloartenol and 24-methylenecycloartanol, progressively accumulate when the olive fruit reaches its final size and veraison begins [13].

Secoiridoids are not soluble in oil and, after the process of mechanical extraction, only a small portion is recovered in the oil, representing the most important microconstituents of virgin olive oils for their health and sensory proprieties [14]. Indeed, olive secoiridoids play a role in the prevention of atherosclerosis and the inhibition of low-density lipoprotein peroxidation [12]. Numerous studies have clearly demonstrated that these compounds exhibit cancer preventive activities [15] and can contribute to the nutritional prevention of osteoporosis [16]. In particular, oleuropein, hydroxytyrosol [15] and oleocanthal [2] have shown effects on human health. The secoiridoids contribute to the quality of olive oil, influencing the oil taste, being responsible for bitter and pungency sensory notes and as primary antioxidants, secoiridoids are involved in oil oxidative stability [5].

Phenolics play a crucial role in the plant response to environmental cues, being the most important defence compounds against defoliating insects [17]. They also affect shoot branching [18] and have been hypothesised to protect cells and prevent fungal penetration into the cambial zone $[19,20]$. Some data support the idea that the resistance to specific pathogens might also be related to certain types of phenolics [21,22]. Oleuropein is responsible for the release of phytoalexins [23], and it is also a multivalent alkylator that functions as an ideal protein cross-linker, exhibiting the strongest activity reported for a plant metabolite, which adversely affects herbivores by decreasing the nutritive value of dietary proteins [24].

To date, secoiridoid metabolism has not been well clarified, but a pathway has been proposed for some Oleaceae species $[25,26]$. Secoiridoid accumulation is a controlled process with expression and composition varying considerably among varieties, tissues, developmental stages and in response to different environmental conditions [27].

The key genes that modulate the synthesis and degradation of secondary compounds in olive fruits have not been characterised, with the exception of a few genes involved in triterpene biosynthesis [28,29], due to the lack of information for the olive genome sequence. The first olive fruit transcriptome data were recently released $[30,31]$, representing an important resource for the identification of genes involved in fruit metabolism.

Functional genetic studies are difficult to perform in perennial woody species due to the lack of efficient protocols for mutagenesis, transformation and in vitro regeneration; therefore, understanding the natural variations for traits of interest represents a valuable tool. In plant science, the integration of gene expression and metabolic data sets is currently being attempted to study metabolic pathways [32].

The aim of this work was to provide further insight into the evolution of the olive fruit phenolic compounds and to identify their major genetic determinants.

\section{Results and discussion}

\section{Concentration of phenolics according to variety and fruit developmental stage}

The cultivars chosen for the analyses represent a high level of variation in the fruit phenolics content, based on a number of studies previously performed on subgroups of these compounds or empirical information, as is the case for Dolce d'Andria and Tendellone, which are traditionally used as table olives and are directly edible without undergoing the debittering process. To our knowledge, our work represents the first attempt to directly compare phenolic profiles of olive cultivars grown under the same environmental conditions. These cultivars, in fact, are cultivated in different regions of Italy, under different climate conditions, soils, water availabilities and agro-techniques that greatly affect the fruit phenolic content. A previous molecular characterisation of these varieties has established that they have originated independently, and no close genetic relationships have been observed among either the high- or lowphenolics cultivars (Baldoni, unpub. data).

The total phenolic content decreased during fruit development, and at the first sampling time (45 DAF), it 
ranged from 50 to $350 \mathrm{mgg}^{-1} \mathrm{dw}$ in the 12 cultivars analysed (Figure 1A). The concentration of the major compounds, such as oleuropein, demethyloleuropein, 3-4 DHPEA-EDA, ligstroside, tyrosol, hydroxytyrosol, verbascoside and lignans, varied among cultivars, some of which had average phenolic levels higher than $150 \mathrm{mg} \mathrm{g}^{-1}$ $\mathrm{dw}$ (Coratina and Rosciola), while others had levels lower than $50 \mathrm{mg} \mathrm{g}^{-1} \mathrm{dw}$ (Tendellone and Dolce d'Andria), and the levels in the remaining cultivars averaged from 60 to $130 \mathrm{mg} \mathrm{g}^{-1} \mathrm{dw}$ (Figures 1 and 2, Additional file 1, Additional file 2 and Additional file 3).

In the high phenolics (HP) cultivars Coratina and Rosciola, oleuropein represented the most abundant compound (up to $82 \%$ of the total), decreasing in concentration after fruit set, as previously observed in other olive varieties [33]. In the low phenolics (LP) cultivars, Tendellone and Dolce d'Andria, the main phenolic compound was not oleuropein, lower than $15 \mathrm{mg} \mathrm{g}^{-1} \mathrm{dw}$

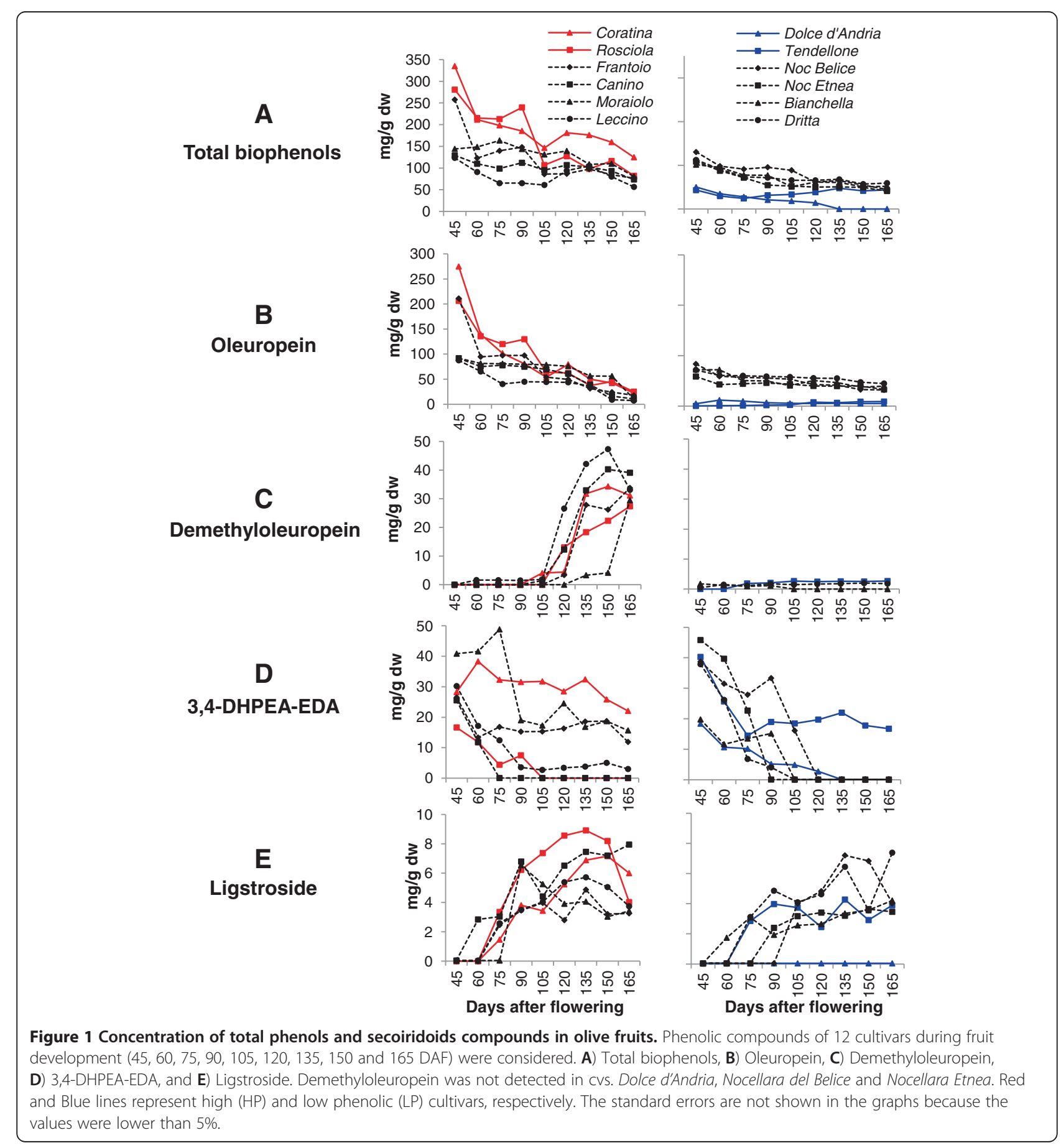




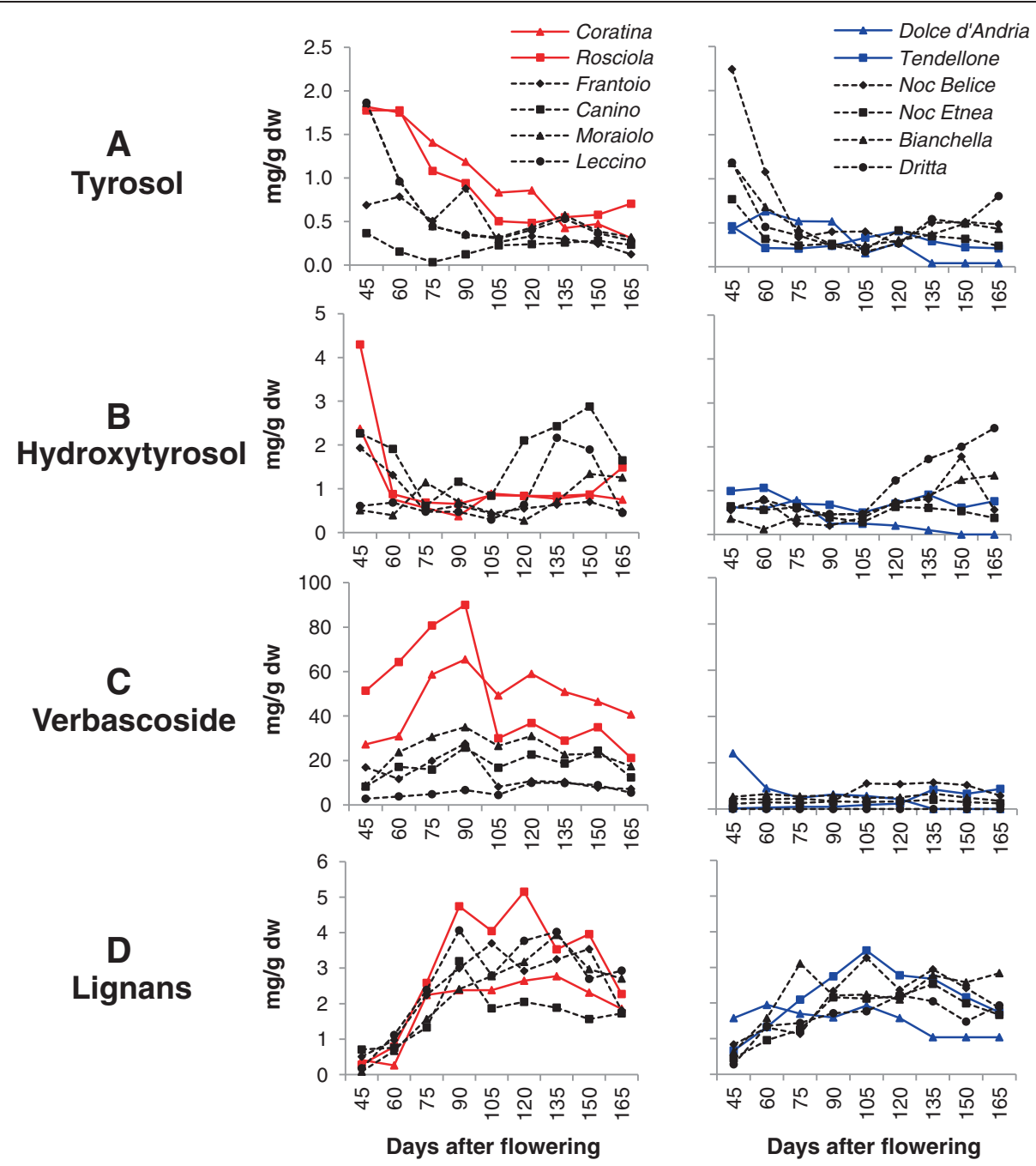

Figure 2 Concentration of phenolic compounds in olive fruits. Phenolic compounds of 12 cultivars during fruit growth (45, 60, 75, 90, 105, 120, 135, 150 and 165 DAF) were considered. A) Tyrosol, B) Hydroxytyrosol, C) Verbascoside, and D) Lignans. Red and Blue lines represent high (HP) and low phenolic (LP) cultivars, respectively. The standard errors are not shown in the graphs because the values were lower than $5 \%$.

during all stages of the maturation process (Figure 1B), but 3-4 DHPEA-EDA, which accounted for up to $94 \%$ of the total phenolic contents at the beginning of fruit development.

Demethyloleuropein showed an opposite trend, accumulating during the last period of fruit development, after 105 DAF, with the highest levels observed in the cvs. Coratina, Rosciola, Frantoio, Canino, Moraiolo and Leccino. In contrast, only trace amounts of this compound (lower than $3 \mathrm{mg} \mathrm{g}^{-1} \mathrm{dw}$ ) were detected in Tendellone, Bianchella and Dritta, and demethyloleuropein expression was completely absent in Dolce d'Andria, Nocellara del Belice and Nocellara Etnea, suggesting a block in the reaction leading to the synthesis or accumulation of this compound (Figure 1C). In HP cultivars the progressive decrease in the oleuropein concentration corresponded with an increase of demethyloleuropein content. The highest concentration of demethyloleuropein was observed at the stages with the lowest concentration of oleuropein. This observation supports the hypothesis of the derivation of demethyloleuropein from the demethylation of oleuropein, as previously envisaged by Obied et al. [1]. In addition, the observation that in LP cultivars and Nocellara del Belice, Nocellara Etnea, Bianchella and Dritta, oleuropein expression and demethyloleuropein content remain constant during fruit development is in accordance with this hypothesis.

The compound 3-4 DHPEA-EDA represented a consistent portion of the total phenolics during the early stages of fruit development in the cvs. Tendellone, Dritta, Nocellara del Belice and Nocellara Etnea (95\%, $31.5 \% 29.7 \%$ and $42.3 \%$, respectively), but the its concentration decreased to values nearing 0 at $90 \mathrm{DAF}$ for most varieties (Figure 1D). Interestingly, in Dolce d'Andria it 
Table 1 Transcripts putatively involved in the secondary metabolism of olive fruits

\begin{tabular}{|c|c|c|c|c|c|}
\hline Pathway & Transcript & $\begin{array}{l}\text { Accession } \\
\text { Number }\end{array}$ & $\begin{array}{l}\text { Length } \\
\text { (bp) }\end{array}$ & Enzymatic function $^{\mathrm{b}}$ & Blast results ${ }^{c}$ \\
\hline \multirow[t]{8}{*}{ MEP pathway } & OeDXS* & $J \times 266162$ & 574 & $\begin{array}{l}\text { 1-deoxy-d-xylulose 5-phosphate synthase } \\
\text { (EC:2.2.1.7) }\end{array}$ & 7.9E-124, 92\% (H. brasiliensis) \\
\hline & OeDXR & JX266164 & 596 & $\begin{array}{l}\text { 1-deoxy-d-xylulose-5-phosphate reductoisomerase } \\
\text { (EC:1.1.1.267) }\end{array}$ & 2.5E-129, 92\% (H. brasiliensis) \\
\hline & OeCDPMES & $J X 266166$ & 927 & $\begin{array}{l}\text { 2-C-methyl-D-erythritol 4-phosphate } \\
\text { cytidyltransferase (EC:2.7.7.60) }\end{array}$ & 4.0E-141, 87\% (S. miltiorrhiza) \\
\hline & OeCDPMEK & JX266168 & 800 & $\begin{array}{l}\text { 4-diphosphocytidyl-2-C-methyl-D-erythritol kinase } \\
\text { (EC:2.7.1.148) }\end{array}$ & $5.1 \mathrm{E}-111,76 \%$ (S. miltiorrhiza) \\
\hline & OeMECPS & $J \times 266170$ & 550 & $\begin{array}{l}\text { 2-C-methyl-D-erythritol 2,4-cyclodiphosphate } \\
\text { synthase (EC:4.6.1.12) }\end{array}$ & $5.8 \mathrm{E}-89,75 \%$ (A. adenophora) \\
\hline & OeHMBPPS & JZ030838 & 178 & $\begin{array}{l}\text { 4-hydroxy-3-methylbut-2-en-1-yl diphosphate } \\
\text { synthase-like (EC 1.17.7.1) }\end{array}$ & $9.0 \mathrm{E}-32,95 \%$ (V. vinifera) \\
\hline & OeHMBPPR* & $J X 266172$ & 1114 & $\begin{array}{l}\text { 4-hydroxy-3-methylbut-2-enyl diphosphate } \\
\text { reductase (EC:1.17.1.2) }\end{array}$ & 0.0, 87\% (S. lycopersicum) \\
\hline & OelPPI & JX266174 & 840 & Isopentenyl diphosphate isomerase (EC:5.3.3.2) & 2.3E-156, 95\% (N. tabacum) \\
\hline \multirow[t]{4}{*}{$\begin{array}{l}\text { Mevalonate pathway } \\
\text { (MVA) }\end{array}$} & OeHMGR & JZ030840 & 214 & $\begin{array}{l}\text { 3-hydroxy-3-methyl glutaryl CoA reductase } \\
\text { (EC:1.1.1.34) }\end{array}$ & 7.6E-40, 89\% (S. nigrum) \\
\hline & OeMVAK & JX266176 & 1152 & Mevalonate kinase (EC:2.7.1.36) & 4.1E-142, 84\% (C. roseus) \\
\hline & OeMVAPK & $J \times 266178$ & 366 & Phosphomevalonate kinase (EC:2.7.4.2) & $6.2 \mathrm{E}-24,67 \%$ (V. vinifera) \\
\hline & OEMVAPPD & $J X 266179$ & 373 & $\begin{array}{l}\text { Mevalonate diphosphate decarboxylase } \\
\text { (EC:4.1.1.33) }\end{array}$ & $1.0 \mathrm{E}-14,85 \%$ (C. roseus) \\
\hline \multirow{9}{*}{$\begin{array}{l}\text { Synthesis of monoterpenic } \\
\text { moiety of secoiridoids }\end{array}$} & OeGES & JX266180 & 1090 & geraniol synthase (EC:4.2.3.-) & 4.0E-166, 76\% (P.dulcis) \\
\hline & OeGE1OH & JX266182 & 1232 & Geraniol 10-hydroxylase (EC:1.14.13.B15) & $0.0,80 \%$ (C. roseus) \\
\hline & OeNDHD* & GQ851611 & 643 & NADH dehydrogenase I (EC:1.6.99.3) & $\begin{array}{l}\text { 1.0E-92, 77\% (P. trichocarpa } x \\
\text { P. deltoides) }\end{array}$ \\
\hline & OeGT* & GQ851612 & 1050 & Glucosyltransferase (EC:2.4.1) & $8.0 \mathrm{E}-126,69 \%$ (N. tabacum) \\
\hline & OeSLS1 & $J \times 266184$ & 907 & Secologanin synthase-like (EC:1.3.3.9) & $1.2 \mathrm{E}-145,67 \%$ (V. vinifera) \\
\hline & OeSLS2 & JX266186 & 1325 & Secologanin synthase-like (EC:1.3.3.9) & $1.1 \mathrm{E}-120,45 \%$ (G. max) \\
\hline & OeSLS3 & JX266188 & 832 & Secologanin synthase-like (EC:1.3.3.9) & $1.8 \mathrm{E}-79,44 \%$ (G. max) \\
\hline & OeSLS4 & $J \times 266190$ & 667 & Secologanin synthase (EC:1.3.3.9) & $1.3 \mathrm{E}-44,38 \%$ (C. roseus) \\
\hline & OeLAMT & $J \times 266191$ & 572 & $\begin{array}{l}\text { S-adenosylmethionine-dependent } \\
\text { methyltransferase (EC:2.1.1.50) }\end{array}$ & $1.9 \mathrm{E}-65,53 \%$ (V. vinifera) \\
\hline \multirow{6}{*}{$\begin{array}{l}\text { Synthesis of phenolic } \\
\text { moieties }\end{array}$} & $O e A D H^{*}$ & GQ851610 & 790 & Arogenate dehydrogenase (EC:1.3.1.43) & 6.0E-70, 84\% (S. pennellii) \\
\hline & OeCuAO* & GQ851613 & 1843 & Copper amine oxidase (EC:1.4.3.21) & $0.0,89 \%$ (R. communis) \\
\hline & OePPO & $J \times 266193$ & 1491 & Polyphenol oxidase (EC:1.10.3.1) & $0.0,60 \%$ (S. indicum) \\
\hline & OETYRD & $J \times 266195$ & 1373 & Tyrosine/dopa decarboxylase (EC:4.1.1.25) & $0.0,78 \%$ (P. somniferum) \\
\hline & OeALDH1 & JX266197 & 793 & Alcohol dehydrogenase (EC:1.1.1.90) & 2.0E-163, 98\% (O. europaea) \\
\hline & OeALDH2 & JX266199 & 487 & Alcohol dehydrogenase class-3 (EC:1.1.1.90) & $1.0 \mathrm{E}-95,93 \%$ (G.max) \\
\hline \multirow{2}{*}{$\begin{array}{l}\text { Phenylpropanoid } \\
\text { biosynthesis }\end{array}$} & OePAL & JX266200 & 1587 & Phenylalanine ammonia-lyase (EC:4.3.1.24) & $0.0,90 \%$ (P. frutescens) \\
\hline & $\mathrm{Oe} 4 \mathrm{CL}$ & JX266202 & 260 & 4-coumarate coenzyme A ligase (EC: 6.2.1.12) & 9.1E-50, 98\% (P. fortunei) \\
\hline \multirow{5}{*}{$\begin{array}{l}\text { Sterol and terpene } \\
\text { biosynthesis }\end{array}$} & OeLS & JZ030839 & 169 & Limonene synthase like (EC: 4.2.3.20) & $6.0 \mathrm{E}-17,67 \%$ (A. thaliana) \\
\hline & OeFPPS & $J \times 266204$ & 1011 & Farnesyl diphosphate synthase (EC:2.5.1.10) & $0.0,89 \%$ (G. uralensis) \\
\hline & OeSQS & JX266206 & 516 & Squalene synthase (EC: $2 \cdot 5.1 .21)$ & 1.2E-111, 95\% (B. monnieri) \\
\hline & OeGGPS & JX266207 & 752 & $\begin{array}{l}\text { Geranylgeranyl pyrophosphate synthase } \\
\text { (EC:2.5.1.29) }\end{array}$ & $2.1 \mathrm{E}-159,90 \%$ (C. roseus) \\
\hline & OeLUPS ${ }^{d}$ & $A B 025343^{d}$ & & & \\
\hline
\end{tabular}


Table 1 Transcripts putatively involved in the secondary metabolism of olive fruits (Continued)

\begin{tabular}{|c|c|c|c|c|c|}
\hline \multirow{2}{*}{$\begin{array}{l}\text { Degradation of } \\
\text { phenolics }\end{array}$} & OeGLU* & HQ585436 & 857 & Beta-1,3-glucosidase (EC:3.2.1.39) & 6.0E-85, 85\% (R. communis) \\
\hline & OePOX* & GQ851609 & 1182 & Peroxidase (EC: 1.11.1.7) & 4.0E-140, $82 \%$ (R. communis) \\
\hline
\end{tabular}

was the only secoiridoid detected at the early stages of fruit development, suggesting that in this cultivar secoiridoid synthesis is not totally blocked and also that this compound could be positioned upstream along the pathway before oleuropein, as reported by Ryan et al. [8].

In all cultivars expressing ligstroside, the concentration of this compound increased with fruit development (Figure 1E). This trend was opposite to that observed for oleuropein, suggesting that this compound might be derived from oleuropein rather than acting as its precursor.

Among phenolics, the tyrosol concentration showed a decreasing pattern from less than $2 \mathrm{mg} \mathrm{g}^{-1} \mathrm{dw}$, whereas the hydroxytyrosol concentration remained below $4 \mathrm{mg} \mathrm{g}^{-1} \mathrm{dw}$, with minor variation among cultivars and fruit developmental stages; notably, a slight increase in hydroxytyrosol concentration was observed after 120 DAF in Canino, Leccino, Dritta and Bianchella (Figure 2A-B, respectively).

Verbascoside, a molecule comprising tyrosol/ hydroxytyrosol and cinnamyl-derivative moieties [1], was detected in consistent amounts (up to $90 \mathrm{mg} \mathrm{g}^{-1} \mathrm{dw}$ ) in Coratina and Rosciola at 90 DAF, while its concentration remained lower than $30 \mathrm{mg} \mathrm{g}^{-1} \mathrm{dw}$ in the other cultivars during all developmental phases (Figure 2C), in accordance with the results of Jemai et al. [34]. These authors detected verbascoside only in earlier maturation stages in the cv. Dhokar. Based on our data, it seems that the two major phenolic compounds of olive fruits, oleuropein and verbascoside, with common tyrosol/hydroxytyrosol moieties, do not show any clear relationship.

The lignans acetoxypinoresinol and pinoresinol (Figure 2D) showed a modest increase in expression during fruit development, with the former being greater than the latter. The strongest difference in the relative content of these compounds was observed in Dolce d'Andria, for which the acetoxypinoresinol contents were approximately ten times higher than those of pinoresinol (Additional file 4).

The metabolic analysis allowed us to select HP and LP cultivars for further transcriptional analyses, as the contrasting phenotypes of these cultivars might reflect differences in the expression of the genes involved in phenolic metabolism.
Identification of transcripts putatively involved in the synthesis of the main secondary metabolites identified from fruit EST datasets

A total of 27 transcripts (Table 1, Additional file 5) identified from the OLEA fruit EST database $[30,35]$ were selected for their putative function within secondary metabolite pathways, considering the major compounds present in olive fruits, such as secoiridoids, phenolics, terpenes and sterols (Figure 3). Although the genes involved in the secoiridoid pathway are unknown because they are synthesised only in a restricted number of species whose genome sequence data are not yet available, it is still possible to predict some of the required enzymatic functions by observing proposed biosynthetic steps. Furthermore, many proposed reactions are similar to those working in parallel pathways in wellcharacterised plant species (e.g., the indole alkaloid pathway studied in Catharanthus roseus) [36-38].

The unigenes showing best tBLASTn scores to protein sequences functionally characterised in other species were selected for subsequent transcriptional analyses. To restrict the number of candidates, only those with the highest levels of expression, deduced by the number of ESTs per contig, were considered. In fact, taking into account that phenolic compounds represent the most abundant secondary metabolites of olive fruit, we assumed that the metabolism should be strongly oriented to their biosynthesis. The E-value and the percentage identity to known proteins have been reported for the selected genes (Table 1, Additional file 5). Although this method has a certain level of uncertainty and further studies are necessary to confirm the proposed functions, the identification of candidate genes considering the similarity to known proteins is a powerful approach, as demonstrated for many other species.

The transcripts were resequenced for the cvs. Coratina and Dolce d'Andria and named according to their putative function.

Among the selected transcripts, some were involved in plastidial 2-C-methyl-d-erythritol 4-phosphate (MEP) and cytosolic mevalonate (MVA) pathways, whereas other candidate transcripts were putatively involved in the synthesis of secoiridoids (monoterpenic and phenolic moieties), other phenolics, terpenoids and sterols (Table 1). 


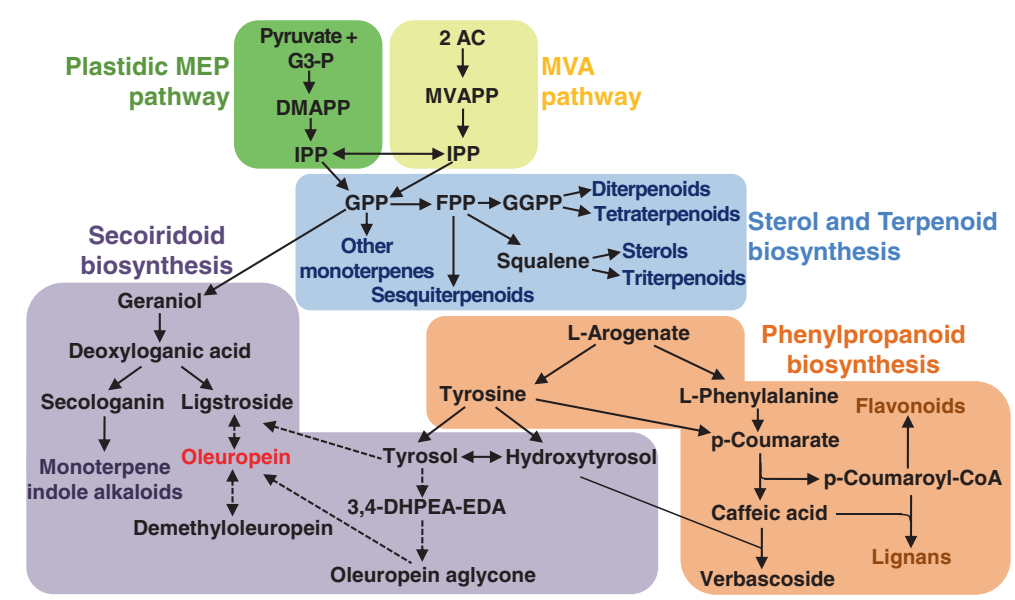

Figure 3 Schematic representation showing the putative biosynthetic pathways of main secondary compounds of olive fruits. G3P: glyceraldehyde 3-phosphate; DMAPP: Dimethylallyl diphosphate; IPP: Isopentenyl diphosphate; AC: Acetyl-CoA; MVAPP: Mevalonate diphosphate; GPP: Geranyl diphosphate; FPP: Farnesyl diphosphate; and GGPP: Geranyl geranyl pyrophosphate. Dotted arrows indicate uncertain biosynthetic steps.

Moreover, an olive gene encoding for a lupeol synthase (LUPS) [Genbank: AB025343], which resulted implicated in triterpenoid biosynthesis in yeast [28], was considered for the expression analyses.

\section{Identification of other transcripts from cDNA-AFLP analysis}

To identify genes that might play a role in secondary metabolism but not included in the fruit EST dataset, a cDNA-AFLP analysis was performed. Comparing the fruit transcriptional profiles of the HP cvs. Coratina and Rosciola and LP cvs. Tendellone and Dolce d'Andria in three developmental stages (45, 90 and 165 DAF) allowed the identification of 93 fragments showing differential expression patterns. For 59 fragments, high quality sequences were obtained and blasted against protein databases. Forty-five transcripts (approximately 76\% of selected sequences) showed significant similarity to proteins with known function. The BLAST results and expression patterns of these transcripts are shown in Additional file 6. The sequences of CDNA-AFLP fragments matched entries in the fruit EST database, with low differences attributable to cultivar allele variations, EST sequence uncertainties, or different genes of the same family.

The results of the Blast2GO analysis allowed the annotation of the expressed sequences according to the terms of three main Gene Ontology vocabularies. The majority of the transcripts encoded for putative proteins with binding or catalytic activities (44\% and 37\%, respectively) (Additional file 7).

For 24 transcripts, the expression profiles were confirmed using semi-quantitative PCR (sqPCR) analysis, and 23 transcripts showed a similar expression pattern to that observed in the cDNA-AFLP analysis (Additional file 8).

Seven transcripts $(17,7 \%)$ were implicated in the synthesis or degradation of secoiridoids (Table 1). The sequences of the transcripts for OeDXS, OeNDHD, OeGT, OeADH, OeCuAO, OeGLU and OePOX were extended to $574,643,1050,790,1843,857$ and 1,182 bp, respectively, using RACE-PCR. The sequences of OeNDHD and OePOX include the complete coding and partial 5' and 3' UTR regions.

The OeDXS sequence exhibited high identity (86\% identity, 92\% similarity) to DXS type II [Genbank: CAD22531] of $M$. truncatula, which is putatively involved in secondary metabolism, and a lower BLASTP score (79\% identity, 86\% similarity) to DXS type I [Genbank:CAD22530], which is proposed to play a role in primary metabolism [39].

\section{Quantitative expression analyses for olive fruit mRNAs}

The expression of the 28 candidate genes identified from EST datasets and the seven selected ones from the cDNA-AFLP analysis, putatively encoding for enzymes involved in secoiridoid, phenolic, terpene and sterol metabolism, was characterised using RT-qPCR to detect a possible correlation with the metabolic data.

The analysis was performed at 45, 90 and 165 DAF using the fruits of HP (Coratina) and LP (Dolce d'Andria) cultivars.

\section{Relative expression of transcripts putatively involved in MEP} and MVA pathways

In higher plants, the five-carbon building blocks of all terpenoids, isopentenyl diphosphate (IPP) and dimethylallyl diphosphate (DMAPP) may derive from the 
plastid-localised MEP pathway and the cytosolic MVA pathway. Enzymes and related genes in both pathways are well known and have been characterised.

The six transcripts putatively involved in the MEP pathway (OeDXS, OeDXR, OeCDPMES, OeMECPS and $O e H M B P P R$ and $O e H M B P P S$ ) (Additional file 9) showed a strong significant decrease in expression from $45 \mathrm{DAF}$ to subsequent developmental stages, whereas $O e C D P$ $M E K$ did not show a significant expression modulation (Figure 4). OeDXS, OeDXR and OeHMBPPR had the strongest differential expression in accordance with their key role in the MEP pathway, which has been proposed in other plant species [40-42]. In particular, our results showed that OeDXS, which is involved in the first step of the MEP pathway, was exclusively expressed in the first developmental stage (45 DAF) in both cultivars. Based on current studies, there are two types of DXS. Type I is constitutively expressed in photosynthetic tissues and is likely involved in the biosynthesis of isoprenoids of primary metabolism, such as carotenoids and
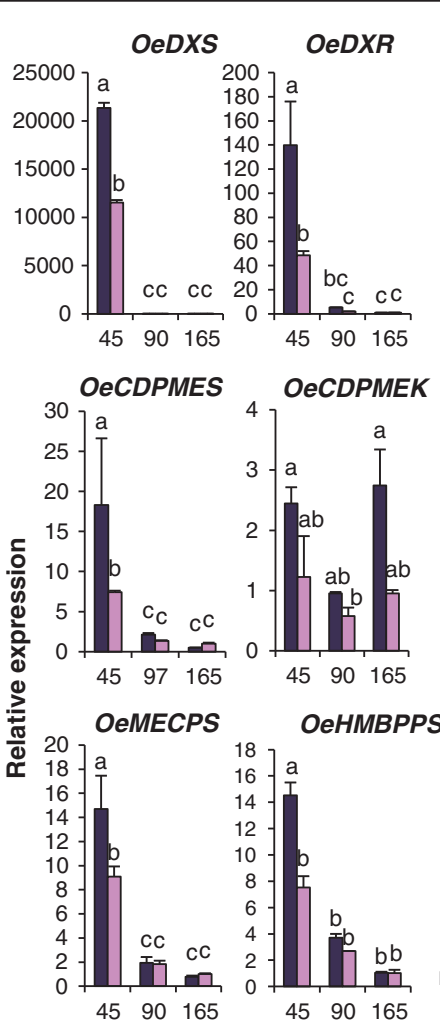

$45 \quad 90 \quad 165$

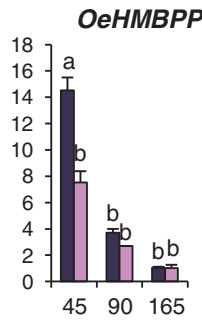

(Carbohydrates)

+ NA

\section{Plastidic MEP Cytosolic pathway MVA pathway}

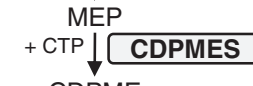

CDPME

+ ATP $\$ CDPMEK

CDPME2P

CMP MECPS

MECP

1 HMBPPS

HMBPP

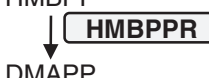

DMAPP

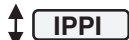

IPP

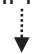

Secoiridoids,

mono-, di-, tetraterpenes

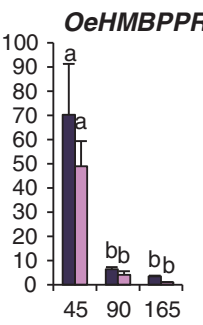

Days after flowering
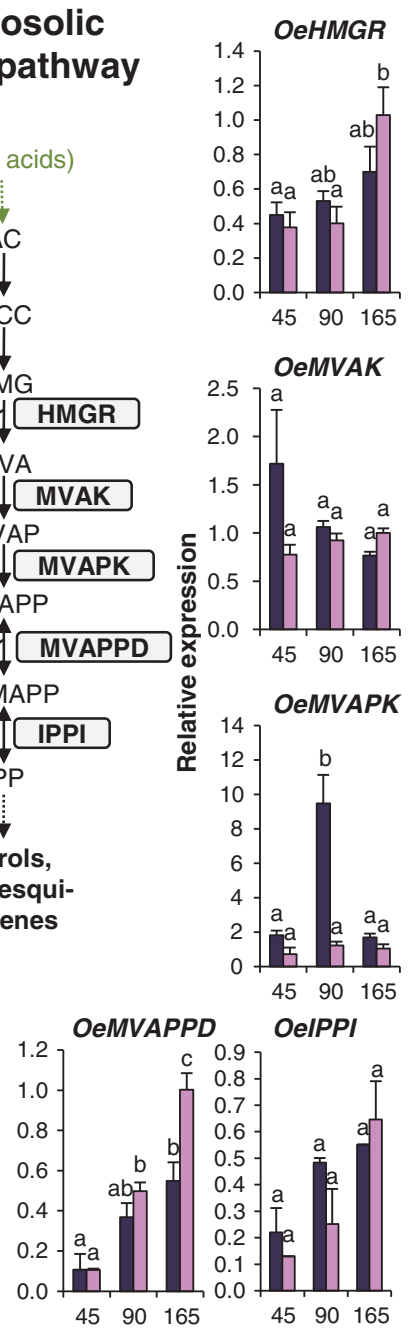

Days after flowering

Figure 4 The expression of genes putatively involved in the MEP and MVA pathways. The mRNA expression of genes putatively involved in 2-C-methyl-d-erythritol 4-phosphate (MEP) and mevalonic acid (MVA) pathways, as determined using RT-qPCR and a schematic representation of these pathways. G3P: glyceraldehyde 3-phosphate; DXP: 1-deoxy-D-xylulose-5-P; DXS: DXP synthase; DXR: DXP reductoisomerase; MEP: 2-C-methyl-D-erythritol-4-P; CDPME: 4-(CDP)-2-C-methyl-D-erythritol; CDPMES: CDPME synthase; CDPMEK: CDPME kinase; CDPME2P: 4-(CDP)-2-C-methyl-D-erythritol-2-P; MECP: 2-C-methyl-D-erythritol 2,4-cyclo-PP; MECPS: MECP synthase; HMBPP: 1-hydroxy-2-methyl-2-(E)-butenyl4-PP; HMBPPS: HMBPP synthase; HMBPPR: HMBPP reductase; DMAPP: Dimethylallyl diphosphate; IPP: Isopentenyl diphosphate; IPPI: IPP delta isomerase; AC: Acetyl-CoA; ACC: Acetoacetyl-CoA; HMG: 3-hydroxy-3-methylglutaryl-CoA; HMGR: HMGC reductase; MVAK: MVA kinase; MVAP: mevalonate phosphate; MVAPK: MVAP kinase; MVAPP: Mevalonate diphosphate; MVAPPD: MVAPP decarboxylase; and DAPP: Dimethylallyl diphosphate. The relative mRNA levels are expressed as $\Delta \Delta C$. Bars $= \pm S E, n=3$. Different letters indicate significant differences between samples, as determined using analysis of variance (Bonferroni's post hoc tests, $\mathrm{P}<0.05$ ). 
phytols, whereas type II DXS seems to be involved in the biosynthesis of isoprenoids for specialised metabolism [43]. It is possible that in the olive there is more than one DXS isoform, and the deduced amino acid sequence of our OeDXS showed similarity to DXS type II, suggesting its involvement in isoprenoids for secoiridoid biosynthesis rather than for primary metabolism. It has been reported that in grape a DXS gene co-localises with a major QTL, affecting monoterpene content [44], and it is putatively responsible for muscat flavour [45]. At 45 DAF, OeDXS, OeDXR, OeCDPMES, OeHMBPPR were significantly more expressed in Coratina than in Dolce d'Andria. OeIPPI, coding for an enzyme involved in both the MEP and MVA pathways for the conversion of IPP to dimethylallyl pyrophosphate (DMAPP), did not show a significant expression difference among the samples (Figure 4).

The four transcripts putatively encoding enzymes involved in the MVA pathway (OeHMGR, OeMVAK, OeMVAPK, OeMVAPPD) showed completely different profiles compared with the MEP transcripts. In particular, OeHMGR and OeMVAK did not display strong differences among the developmental stages or varieties, except for a weak expression increase at 165 DAF in cv. Coratina (Figure 4).

Two isoforms of the enzyme HMGR were identified in Coffea arabica fruits and transcripts of isoform CaHMGR1 were expressed only at the initial stages of fruit development, while the isoform CaHMGR2 was constitutively expressed [46]. It is also possible that these two isoforms are expressed in the olive fruit, and it is likely that we have identified the constitutive form.

Coratina OeMVAPK was more expressed at fruit pit hardening (90 DAF), whereas in Dolce d'Andria, it was expressed at the same level in all three developmental stages. OeMVAPPD mRNA increased during fruit development and was always more highly expressed (up to 13-fold) in Coratina than in Dolce d'Andria.

Unlike transcripts involved in the MVA pathway, the expression patterns of transcripts involved in the MEP pathway, in accordance with secoiridoid, decrease during fruit development, suggesting that the MEP pathway is also present in olives and might contribute to the terpenoid portion of secoiridoids. These results are consistent with those reported in other plant species, where MEP and MVA pathways produce different terpenoid classes. In fact, it is generally accepted that geranyl diphosphate (GDP) and geranylgeranyl diphosphate (GGDP), deriving from the MEP pathway, are used in plastids as substrates for monoterpene, diterpene and tetraterpene synthesis, whereas farnesyl diphosphate (FDP), obtained from the MVA pathway in the cytosol, is involved in triterpene and sesquiterpene biosynthesis [47]. However, cross talk between these two different
IPP biosynthetic pathways has been documented, and the relative contribution of each pathway to the biosynthesis of the various classes of terpenes remains uncertain [48]. In olive, only a contrasting report has been published [49], suggesting the involvement of the MVA pathway in secoiridoid formation in the Oleaceae family, but neither enzymatic nor molecular data were provided.

\section{Relative expression of transcripts putatively involved in biosynthesis of the terpenic moiety of secoiridoids}

Biosynthetic steps leading to formation of the terpenic and phenolic portions of secoiridoids are still not well clarified and, as a consequence, enzymes involved in these pathways remain uncertain.

Transcripts putatively involved in the synthesis of the terpenic portion of secoiridoids (Additional file 10), OeGES, OeGE1OH, OeNDHD, OeGT, OeLAMT, and the four transcripts putatively coding for secologanin synthase, OeSLS1, OeSLS2, OeSLS3 and OeSLS4, were analysed (Figure 5A). Their expression dramatically decreased from 45 to $165 \mathrm{DAF}$, with the largest variation occurring between 45 and 90 DAF, in accordance with the decay of oleuropein concentration observed during fruit ripening. Significant differences between varieties were also observed for OeGE1OH and OeNDHI, which appeared to be more highly expressed in Coratina than in Dolce d'Andria at 45 DAF.

GES is a monoterpene synthase involved in the synthesis of geraniol [50], and $\mathrm{GE} 10 \mathrm{H}$ is a cytochrome P450 monooxygenase that hydroxylates the monoterpenoid geraniol at the $\mathrm{C}-10$ position to generate 10hydroxygeraniol. This enzyme was reported to be involved in the biosynthesis of iridoid monoterpenoids and several classes of monoterpenoid alkaloids observed in a diverse range of plant species [51]. Feeding experiments on Syringa and Fraxinus (other genera within the Oleaceae family) showed that the biosynthesis of oleoside-type secoiridoids proceeds via iridodial $[25,26,52]$, which presumably originates from geraniol and 10hydroxygeraniol [1,49,53] (Additional file 10). Interestingly, it has been recently shown using in vitro enzymatic assays that the same OeGES1 that we have analysed is involved in the synthesis of geraniol [54], demonstrating the effectiveness of our approach in identifying candidate transcripts for phenolics biosynthesis.

The $\mathrm{G} 10 \mathrm{H}$ of C. roseus $(\mathrm{CrG10H})$ also catalyses the 3'hydroxylation of naringenin to produce eriodictyol with a catalytic activity efficiency that is 10 times lower compared to geraniol hydroxylation. These studies demonstrated that $\mathrm{G} 10 \mathrm{H}$ plays an important role in the biosynthesis of flavonoids, in addition to its previously described role in the metabolism of terpenoids [51]. Based on these studies, we cannot exclude that OeG10H 


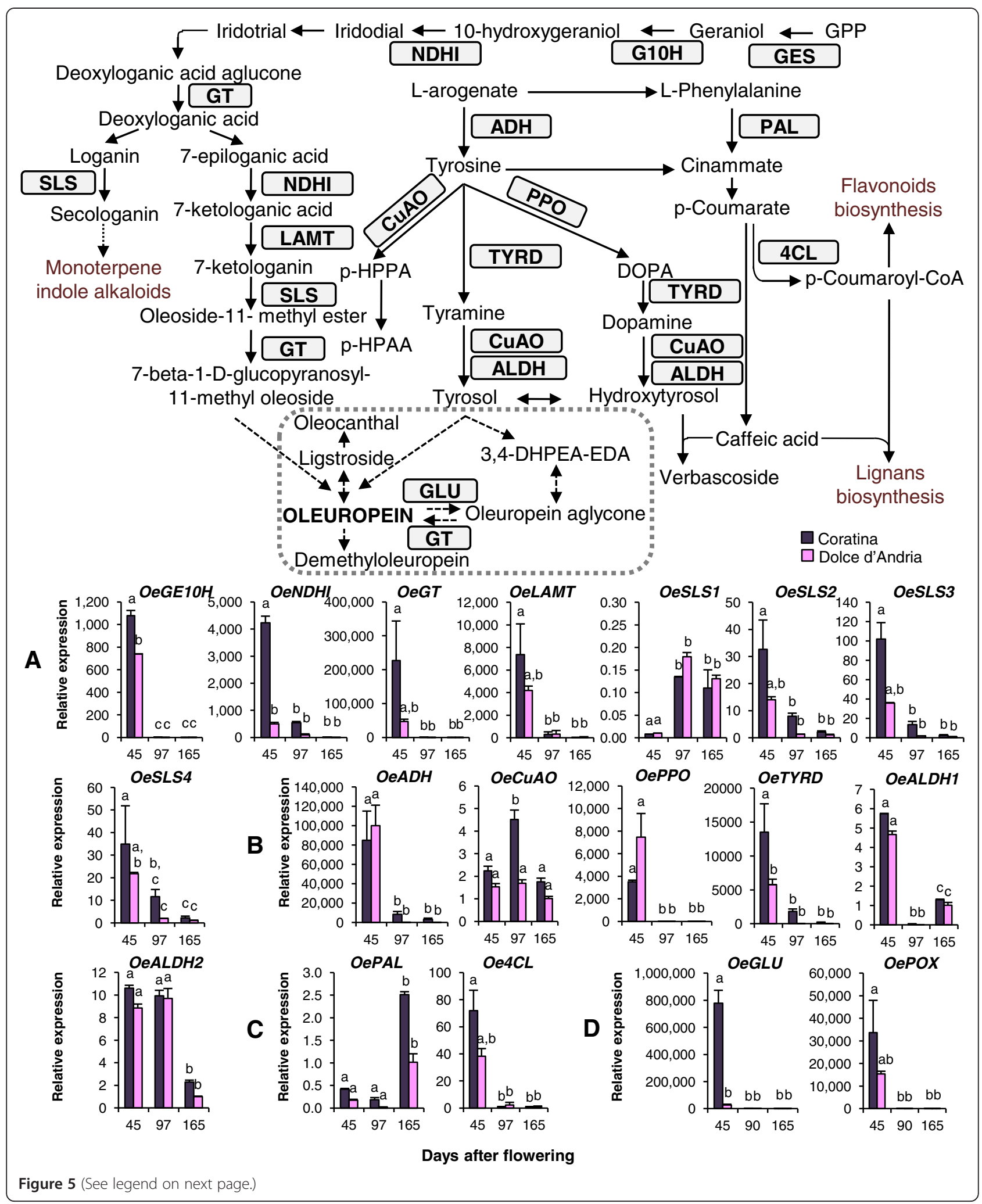


(See figure on previous page.)

Figure $\mathbf{5}$ The expression of genes putatively involved in the metabolism of secoiridoids and other phenolics compounds. The mRNA expression of genes putatively involved in the biosynthesis of terpenic (A) and phenolic moieties (B) of secoiridoids, in the biosynthesis of phenylpropanoids (C), in the degradation of phenolic compounds, and a schematic representation of their metabolism. The mRNA level was determined using RT-qPCR. GES: geraniol synthase; G10H: Geraniol 10-hydroxylase; NDHI: NADH dehydrogenase l; GT: SLS: Secologanin synthase; LAMT: Loganic acid methyltransferase; ADH: Arogenate dehydrogenase; CuAO: Copper amine oxidase; $p$-HPPA: $p$-hydroxyphenylpyruvic acid; $p$ HPAA: p-hydroxyphenylacetic acid; TYRD: Tyrosine/dopa decarboxylase; ALDH: Alcohol dehydrogenase; PPO: Polyphenol oxidase; PAL:

Phenylalanine ammonia-lyase; and $4 \mathrm{CL}$ : 4-coumarate coenzyme A ligase. The relative mRNA levels are expressed as $\Delta \Delta C \mathrm{Ct}$. Bars $= \pm \mathrm{SE}, \mathrm{n}=3$. Different letters indicate significant differences between samples as determined using analysis of variance (Bonferroni's post hoc tests, $\mathrm{P}<0.05$ ). Grey dotted box includes the secoiridoids compounds. Dotted arrows indicates uncertain biosynthetic steps.

might also be involved in flavonoid biosynthesis in the olive.

Despite the fact that NDHI and GT are enzymes required in different pathways, the strong differential expression of OeNDHI and OeGT in LP and HP varieties and during different stages of fruit development, suggested a putative role for these transcripts in secoiridoid metabolism. These enzymes might play a role in various steps of the pathway. NDHI might work in both, the conversion of iridotrial to deoxyloganic acid aglucone and the conversion of 7-epi-loganic acid to 7ketologanic acid. GTs are required for terpenic and phenolic fractions, transferring glucosylic groups to deoxyloganic aglucones for the formation of deoxyloganic acid, the conversion of oleoside 11-methyl ester to 7-ß-1-D-glucopyranosyl 11-methyloleoside and catalysing the formation of oleuropein from oleuropein aglycone, which is the last step of the pathway (Additional file 10).

The position of 3,4-DHPEA-EDA along the secoiridoid pathway remains controversial. It has been considered either as a derivative of oleuropein, produced by its enzymatic degradation by endogenous $\beta$-glucosidases [14] (Additional file 2), or as the intermediate compound of an alternative biosynthetic pathway leading to oleuropein formation $[1,8,55]$. The high level of expression of genes encoding enzymes promoting the conversion of 3,4-DHPEA-EDA to oleuropein, such as OeGT, during the early stages of fruit development in the HP cv. Coratina supports the hypothesis of 3,4-DHPEA-EDA as an oleuropein precursor. This hypothesis finds a further confirmation in the observation that 3,4-DHPEA-EDA is the only secoiridoid detected in cv. Dolce d'Andria at the early stages of fruit development, suggesting that a downstream block may prevent the formation of the other secoiridoids.

In O. europaea both epoxides of secologanin and secoxyloganin could be precursors of oleuropein $[49,53]$. The oxidation required for the conversion of 7 ketologanin to oleoside-11- methyl ester is similar to the mechanism taking place for the conversion of loganin, an epimer of 7-ketologanin, to secologanin. Therefore, we assumed that the gene encoding this enzymatic function might have high sequence similarity with the secologanin synthase (a cytochrome P450 enzyme). Moreover, the role played by loganin and secologanin in secoiridoid biosynthesis in Olea europaea remains controversial, and it cannot be excluded that these compounds might be intermediates of secoiridoid biosynthesis [52], further supporting the involvement of a secologanin synthase.

In the fruit EST database, we identified four transcripts (OeSLS1, OeSLS2, OeSLS3, OeSLS4) showing high similarities to secologanin synthase. Using RT-qPCR analyses, OeSLS2, OeSLS3, OeSLS4 showed a pattern similar to the other transcripts that were included in the secoiridoid synthesis, supporting the hypothesis of their involvement in this pathway. However, OeSLS1 showed a low level of expression at the first sampling, which increased later. This result could be explained by the putative involvement of OeSLS1 in the biosynthesis of other secondary compounds, such as terpene indole alkaloids, as reported in other plant species [56], rather than playing a major role in oleuropein synthesis.

The methylation of 7-ketologanic acid might be catalysed by an enzyme similar to loganic acid Omethyltransferase (LAMT), which converts loganic acid to loganin (epimers of 7-ketologanic acid and 7-ketologanin, respectively), as indicated by the functional characterization in C. roseus (CrLAMT), showing that this enzyme exhibits high specificity for the loganic acid substrate [38]. Therefore, we identified a putative olive homolog (OeLAMT) of the LAMT gene.

\section{Relative expression of transcripts putatively involved in phenolics biosynthesis}

The phenolic moiety of secoiridoids is presumably derived from tyrosine and proceeds through tyrosol $[1,8]$ (Additional file 11). In most plants, tyrosine is synthesised from arogenate decarboxylated by arogenate dehydrogenase $(\mathrm{ADH})$ [57,58], and hydroxytyrosol is synthesised from tyrosine through DOPA and dopamine. Recently, the biosynthesis of hydroxytyrosol was clarified in Olea europaea using cultured cells [59]. Another pathway for the tyrosol formation has been reported in other plant species, where tyrosol might be produced 
from a $p$-coumaric acid precursor, which is derived primarily from phenylalanine [60]. However, in olive, the presence of this alternative pathway has never been demonstrated. Instead, two alternative routes from tyrosol to oleuropein have been proposed: one considering ligstroside as direct oleuropein precursor [49] and the other proceeding via oleuropein aglycone [8] (Additional file 11). Alternative biosynthetic pathways are proposed for verbascoside, e.g., from tyramine via dopamine or from tyrosol via hydroxytyrosol [59].

A schematic representation of the putative olive phenolics biosynthetic pathway is proposed in Figure 5, according to the previous findings discussed above. Based on these findings, we searched for transcripts putatively involved in the biosynthesis of the phenolic portion of secoiridoids and other related phenolic compounds, such as tyrosol, hydroxytyrosol and verbascoside.

We selected and analysed the following genes that might function in the considered pathway: OeADH, OeCuAO, OePPO, OeTYRD, OeALDH1 and OeALDH2. $\mathrm{ADH}$ decarboxylates the arogenate producing tyrosine [61]. CuAO deaminates various compounds with biologically active amines producing their corresponding aminoaldehydes, $\mathrm{H}_{2} \mathrm{O}_{2}$ and $\mathrm{NH}_{3}$ [62]. PPO catalyses the $o$-hydroxylation of monophenols to $o$-diphenols, and it might be involved in different steps of phenolics metabolism [63]. An enzyme similar to a tyrosine/dopa decarboxylase (TYRD) is required for both the conversion of tyrosine in tyramine and DOPA in dopamine $[64,65]$. The conversion of both tyramine to tyrosol and dopamine to hydroxytyrosol requires an amino-oxidase (AO) and an alcohol dehydrogenase (ALDH) $[59,60]$.

The expression profiles of four of the six genes (OeADH, OePPO, OeTYRD and OeALDH1) correlated with the secoiridoid content, decreasing during fruit development, similarly to the genes involved in the terpenic moiety (Figure 5B). mRNAs of OeADH, OePPO and OeTYRD were exclusively present at $45 \mathrm{DAF}$, and $O e A L D H 1$ and $O e A L D H 2$ were more highly expressed at the first sampling and strongly decreased with fruit development. OeTYRD was more expressed in Coratina at 45 DAF compared to Dolce d'Andria. Only OeCuAO was more highly expressed at 90 DAF, decreasing at 165 DAF in Coratina, whereas OeCuAO expression remained constant during development in Dolce d'Andria.

In phenylpropanoid metabolism, two key genes have been investigated: $P A L$ and $4 C L$. They are involved in the synthesis of large groups of compounds, such as flavonoids, lignans and verbascoside. The expression of OePAL increased at 165 DAF in both cultivars, whereas Oe4CL showed the highest expression only at the first sampling in both cultivars (Figure 5C). The strong increase of OePAL mRNA levels at 165 DAF is consistent with the accumulation of anthocyanins and the change in fruit colour to purple-black that occurs in this phase [66]. The opposite trend observed for Oe4CL did not correlate with the content of lignans and flavonoids. In fact, the concentration of lignans first increased and then decreased during fruit growth in all cultivars, whereas the main flavonoids, rutin and luteolin 7-Oglucoside, are reported to increase during olive fruit ripening [67]. It is possible that Oe4CL might not be associated with lignans or flavonoid formation but with that of unique phenylpropanoid end-products, as reported for some species, such as Arabidopsis, aspen and soybean [68].

\section{Relative expression of transcripts putatively involved in phenolics degradation}

The expression of putative OeGLU and OePOX orthologs was analysed. GLU and POX enzymes are involved in phenolic degradation; moreover, GLU plays a role in the formation of oleuropein and ligstroside derivatives [5] (Additional file 2).

OeGLU was almost exclusively expressed during the early stages of fruit development (45 DAF) of cultivar Coratina, whereas OePOX was almost exclusively expressed at 45 DAF in both cultivars (Figure 5D). These profiles are similar to those observed for transcripts putatively involved in secoiridoid synthesis. These results confirm the role of these enzymes in processes that lead to the decrease in phenolic concentration observed at 90 and $165 \mathrm{DAF}$, and their expression might be downregulated whenever a lower availability of oleuropein, their main substrate, occurs [69]. A similar mechanism might explain the lower expression observed for both types of transcripts in the LP cultivar, where the lack of oleuropein could be due to differences in the regulation of enzymes involved in its biosynthesis rather than in its degradation.

\section{Relative expression of transcripts putatively involved in terpenoid and sterol biosynthesis}

The expression of OeLS, putatively leading to the synthesis of limonene, an important volatile monoterpene, showed a strong variation during fruit development. In particular, it was almost exclusively detected at the last sampling in both analysed cultivars (Figure 6A). This pattern is perfectly consistent with the limonene content in unripe and ripe fruits [70].

OeFPPS and OeSQS mRNA levels, putatively coding for two key enzymes leading to the synthesis of squalene, were also analysed, and their expression reached a peak at 90 DAF in Coratina (Figure 6B). It is noteworthy that important compounds are synthesised from squaleneoxide at the onset of fruit development, such as maslinic 


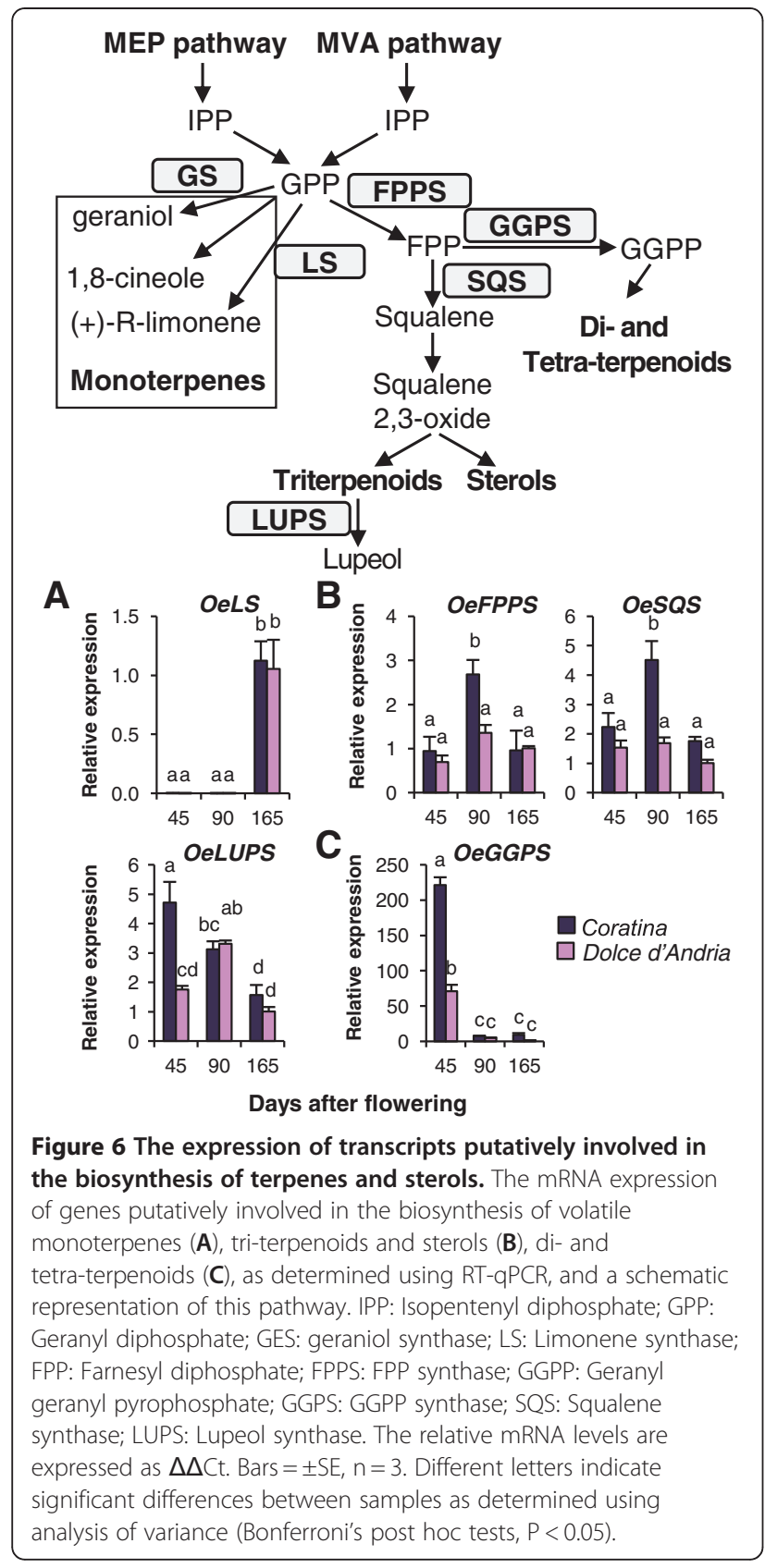

and oleanolic acids and $\alpha$ - and $\beta$-amyrins [13]. The expression profiles observed for OeFPPS and OeSQS of Coratina were consistent with these findings. In contrast, differences among developmental stages were not observed in Dolce d'Andria.

The expression profile in developing fruits of the gene for lupeol synthase, catalysing the formation of triterpenic lupeol and functionally characterised in yeast [28], has never been analysed. Our OeLUPS showed a different mRNA profile between the two cultivars at 45 DAF (Figure 6B), which was more highly expressed in Coratina than in Dolce d'Andria, and, in the last two stages, they shared the same pattern. These results could be explained by a different accumulation of squalene and triterpenes during fruit development in Dolce d'Andria; however, the accumulation of these compounds in this variety has not yet been investigated.

The mRNA levels of GGPS, catalysing the synthesis of geranyl geranyl pyrophosphate (GGPP), an important intermediate for diterpenes and carotenoids formation, were higher at 45 DAF compared with the other developmental stages in both cultivars, and, at the same stage, the expression was higher in Coratina (Figure 6C). Our results are consistent with the higher accumulation of carotenoids during the first stages of olive fruit development [71].

\section{Conclusions}

Our study represents an effort to characterise the transcriptional profile of candidate genes putatively involved in secondary metabolism in olive fruits by assessing the content of major phenolic compounds in a comprehensive number of cultivars at different fruit developmental stages. These methods allowed us to identify candidate genes for secondary metabolites.

We observed a strong variation in overall metabolite content, in the level of specific compounds and in the accumulation trends among genotypes and fruit developmental stages. Coratina and Rosciola were characterised by the highest oleuropein concentration at 45 DAF. In contrast, Dolce d'Andria and Tendellone showed the lowest content at all stages. Interestingly, we identified genotypes characterised by the complete absence of other compounds. In particular, Nocellara del Belice, Dolce d'Andria and Nocellara Etnea did not accumulate demethyloleuropein, and Dolce d'Andria was the only variety showing undetectable amounts of all secoiridoids, excepting 3,4-DHPEA-EDA at the early stages of fruit development. These findings might result from a block in the enzymatic steps leading to the biosynthesis of these compounds.

The evidence of an accumulation of ligstroside occurring when oleuropein decreases, leaves space to the hypothesis that ligstroside may derive from oleuropein instead of the contrary.

The observed differences in the phenolic profiles among the cultivars might reflect great variability in the modulation of their biosynthesis and accumulation. This variability can be exploited in breeding programmes to increase the fruit composition of important phenolic compounds.

The recent development of EST datasets for olive fruits allowed the extrapolation of gene information using sequence-similarity-based approaches. We used protein sequences that were previously characterised or had an assigned function in other species. The high level of similarity detected allowed us to predict the enzyme 
classes and, with some degree of approximation, the substrate specificity. As a complementary approach, the cDNA-AFLP analysis proved to be an efficient technique for the isolation of differentially expressed transcripts without any sequence similarity assumption.

Based on these approaches, 36 transcripts were identified and their expression profiles were characterised and associated with corresponding metabolite profiles.

The strong correlation observed between the content of specific metabolites during fruit development and the expression of transcripts putatively involved in their biosynthesis, suggests that metabolite content is regulated at transcriptional level and strengthens the involvement of the candidate genes in the proposed pathways. The differences observed in the expression of some genes between Coratina and Dolce d'Andria might indicate a different regulation of the transcripts involved in the secondary metabolism among olive genotypes.

In particular, the levels of most of transcripts putatively involved in the biosynthesis of secoiridoids (both terpenic and phenolic moieties) showed a strong decrease during fruit development, according to the decrease in oleuropein concentration at the same stages, and many genes involved in secoiridoid pathway were more highly expressed in HP cv. Coratina than in LP cv. Dolce d'Andria.

We observed a correlation between the MEP pathway and secoiridoid transcriptional profiles, supporting the hypothesis that this pathway, rather than the MVA pathway, primarily contributes to secoiridoid biosynthesis.

Interestingly, some key genes of monoterpenoid and phenolic biosynthesis, such as OeDXS, OeGES, OeG1OH and $O e A D H$, were exclusively expressed in the earliest sampling, when the highest secoiridoid concentration was detected. These genes might regulate the accumulation of these compounds during fruit development.

A strong correlation between metabolic and transcriptional data was also identified for the biosynthesis of limonene and GGPP. OeLS was almost exclusively expressed at the developmental stage when the highest level of this compound was detected. OeGGPPS, putatively involved in carotenoid biosynthesis, was expressed only in young fruits when the highest levels of these compounds occur, as reported in other studies.

These data provide useful information for functional genetic studies of this crop species and for the identification of functional markers related to the accumulation of compounds and metabolites affecting the nutraceutical and organoleptic properties of olive fruits and oil.

\section{Methods}

\section{Plant material}

Based on previous information on the phenolic profile of their oils, the following cultivars were chosen, putatively representing a high level of variation in fruit phenolics content: Bianchella, Canino, Coratina, Dolce d'Andria, Dritta, Frantoio, Leccino, Moraiolo, Nocellara del Belice, Nocellara Etnea, Rosciola and Tendellone. Fruits used for the phenolic composition and cDNA-AFLP analyses were harvested from 45 to 165 days after full bloom (DAF) every 15 days from plants of an olive cultivar collection at the experimental farm of the CRA-OLI (Collececco, Spoleto, Perugia) in central Italy $\left(42^{\circ} 48^{\prime} 48^{\prime \prime} \mathrm{N}\right.$, $12^{\circ} 39^{\prime} 15^{\prime \prime} \mathrm{E}, 356 \mathrm{~m}$ above sea level). The phenological stage of the fruits at sampling dates was recorded. Plants were grown under the same environmental and agronomical conditions. To avoid possible effects of different levels of water availability on the phenolic content among trees, their water status was periodically monitored and occasional irrigation was applied as needed to maintain all plants at similar values of pre-dawn water potential during the sampling period (data not shown). Immediately after harvesting, the olive fruits were frozen in liquid nitrogen and stored at $-80^{\circ} \mathrm{C}$ until further analysis.

\section{Evaluation of phenolic compounds}

The extraction of phenolic compounds was performed according to Servili et al. [72], with few modifications, Briefly, $3 \mathrm{~g}$ of olive fruit mesocarp and exocarp were homogenised using a Homogeniser A/S N (Foss Electric, Denmark) in a 100-ml solution of methanol/water $80: 20 \%$, followed by two further homogenisation in $50 \mathrm{ml}$ of methanol/water 80:20 using an Ultra-Turrax T 25 (IKA, Staufen, Germany). After methanol evaporation in vacuum under a nitrogen flow at $37^{\circ} \mathrm{C}$, solid-phase extraction (SPE) was performed to separate the phenolics from the aqueous extract. During the SPE, a 900-mg Extraclean high load $\mathrm{C}_{18}$ cartridge (Alltech Italia s.r.l., Sedriano, Italy) was loaded with $1 \mathrm{ml}$ of olive extract using $50 \mathrm{ml}$ of methanol as the eluting solvent.

The HPLC analysis was performed according to Selvaggini et al. [73], using a Spherisorb ODS-1 $250 \mathrm{~mm} x$ $4.6 \mathrm{~mm}$ column with a particle size of $5 \mu \mathrm{m}$ (Phase Separation Ltd., Deeside, UK).

The phenolic separation was performed using semipreparative high-performance liquid chromatography (HPLC) analysis with a $9.4 \mathrm{~mm}$ i.d. 500-mm Whatman Partisil 10 ODS-2 semipreparative column; the mobile phase was $0.2 \%$ acetic acid in water $(\mathrm{pH} 3.1)(\mathrm{A}) /$ methanol (B) at a flow rate of $6.5 \mathrm{~mL} / \mathrm{min}$ and phenol detection was performed using a diode array detector (DAD). The purity of all compounds obtained from direct extraction was tested using HPLC, and their chemical structure was verified using nuclear magnetic resonance (NMR) with the same operative conditions reported in previous studies [72]. The following phenolic compounds were considered: oleuropein, demethyloleuropein, 3,4- 
DHPEA-EDA, ligstroside, tyrosol, hydroxytyrosol, verbascoside and lignans.

\section{Identification of mRNAs putatively involved in the metabolism of phenolic compounds}

Sequences derived from the OLEA EST database [35], a collection of over 102,000 Olea europaea L. fruit EST reads generated through 454 massive sequencing technology [30], were analysed to identify transcripts putatively involved in the pathways of terpenoids, phenolics and other secondary metabolite synthesis. Amino acid sequences of genes involved in the pathways of interest and published in the Kyoto Encyclopaedia of Genes and Genomes (KEGG) databases [74,75] were used to search olive homologs in the OLEA EST database using basic local alignment (tBLASTn). For those genes represented using multiple unique transcripts, only the largest contig and/or that representing the highest number of singletons was used for the analysis. Sequences obtained from this collection include candidates for the isoprenoid (both MVP and MEP pathways), phenylpropanoid, terpene (monoterpenes, secoiridoids, diterpenes and triterpenes), phenol, sterol, lignan and flavonoid biosynthesis, leading to the main secondary metabolites present in the olive fruit. All transcripts of interest were resequenced in the HP and LP cultivars, Coratina and Dolce D'Andria, respectively, using gene-specific primers (Additional file 12).

\section{CDNA-AFLP analysis}

Total RNA was isolated from fruit mesocarp and exocarp using the RNeasy Plant Mini Kit (Qiagen), and contaminating genomic DNA were removed with DNase I (Qiagen) treatment. Samples of two HP (Coratina and Rosciola) and two LP (Tendellone and Dolce d'Andria) cultivars, at three samplings (45, 90 and 165 DAF), were considered.

PolyA RNA was isolated from approximately $50 \mu \mathrm{g}$ of total RNA using oligo dT Dynabeads (Invitrogen). All purified mRNA was used to synthesise first strand cDNA using SuperScript III (Invitrogen) according to the manufacturer's 18-bp oligo $\mathrm{d}(\mathrm{T})$ protocol. Doublestranded (ds) cDNA was synthesised by incubating the first-strand product for $2 \mathrm{~h}$ at $16^{\circ} \mathrm{C}$ with $30 \mathrm{U}$ of DNA polymerase I (Invitrogen) and $3 \mathrm{U}$ of RNase $\mathrm{H}$ (Invitrogen) in a reaction mixture containing $20 \mathrm{mM}$ Tris $-\mathrm{HCl}$, $75 \mathrm{mM} \mathrm{KCl}, 10 \mathrm{mM}\left(\mathrm{NH}_{4}\right)_{2} \mathrm{SO}_{4}, 5 \mathrm{mM} \mathrm{MgCl} 2$ and $1 \mathrm{mM}$ DTT. Subsequently, a $10-\mu \mathrm{l}$ aliquot of each sample was assessed on a 1\% agarose gel, and a clear DNA smear was visible between 500 and $4,000 \mathrm{bp}$. The samples were purified using a phenol/chloroform procedure and quantified using a spectrophotometer at a wavelength of $260 \mathrm{~nm}$.
The cDNA-AFLP procedure was conducted according to Bachem and coworkers [76], with some modifications. A total of $500 \mathrm{ng}$ of cDNA was digested in a 50- $\mu \mathrm{l}$ volume using $10 \mathrm{U}$ of MseI and $20 \mathrm{U}$ of EcoRI for $2 \mathrm{~h}$ at $37^{\circ} \mathrm{C}$. The digestion mix was ligated to $5 \mathrm{pmol}$ of EcoRI adapter and 50 pmol of MseI adapter using $68 \mathrm{U}$ of T4 Ligase (New England Biolabs). The reaction was performed for $2 \mathrm{~h}$ at $37^{\circ} \mathrm{C}$. The $\mathrm{PCR}$ reaction solution $(50 \mu \mathrm{l})$ for preamplification contained $10 \mu \mathrm{l}$ of the digestion mix, $75 \mathrm{ng}$ of each primer constructed using the adaptors, $0.2 \mathrm{mM}$ dNTP mix, $1.5 \mathrm{mM} \mathrm{Mg}^{2+}$ and $1 \mathrm{U}$ of Taq polymerase (Invitrogen). The PCR reaction was conducted using the following conditions: $94^{\circ} \mathrm{C}$ for $2 \mathrm{~min}$, 25 cycles at $94^{\circ} \mathrm{C}$ for $30 \mathrm{sec}, 56^{\circ} \mathrm{C}$ for $1 \mathrm{~min}$ and $72^{\circ} \mathrm{C}$ for $1 \mathrm{~min}$, followed by $72^{\circ} \mathrm{C}$ for $7 \mathrm{~min}$. The selective amplification was performed using $5 \mu \mathrm{l}$ of preamplification product diluted 1:10, $60 \mathrm{ng}$ of each selective primer, $1.5 \mathrm{mM} \mathrm{Mg}^{2+}, 0.2 \mathrm{mM}$ dNTP mix and $1 \mathrm{U}$ of Taq polymerase in a $20 \mu \mathrm{l}$ total reaction volume. A total of 20 combinations of the selective primers containing 3 and 2 selective bases for the primer were designed using the EcoRI and MseI adapters, respectively, and the Eco primers were labelled with fluorescein. Touch-down PCR was performed using the following cycling parameters: $94^{\circ} \mathrm{C}$ for $2 \mathrm{~min}, 13 \mathrm{cycles}$ at: $94^{\circ} \mathrm{C}$ for $30 \mathrm{sec}, 65^{\circ} \mathrm{C}\left(-0.7^{\circ}\right.$ $\mathrm{C} /$ cycle) for $30 \mathrm{sec}$ and $72^{\circ} \mathrm{C}$ for $1 \mathrm{~min}, 23$ cycles at $94^{\circ} \mathrm{C}$ for $30 \mathrm{sec}, 56^{\circ} \mathrm{C}$ for $30 \mathrm{sec}$ and $72^{\circ} \mathrm{C}$ for $1 \mathrm{~min}$, followed by $72^{\circ} \mathrm{C}$ for $7 \mathrm{~min}$. The selected PCR product $(5.7 \mu \mathrm{l})$ was denatured at $96^{\circ} \mathrm{C}$ for $12 \mathrm{~min}$ and separated on a $4 \%$ denatured polyacrylamide gel, pre-run at 6 Watts for $10 \mathrm{~min}$ and run at $125 \mathrm{Watts}$ for $1.75 \mathrm{~h}$ at $50^{\circ} \mathrm{C}$ in Genomyx GX 100 (Beckman Instruments, Fullerton, CA). The gel was scanned, and the separated fragments were visualised on a gel using fluorescent emission. Any differentially expressed fragment was excised from the gel, treated in a speed vacuum for $30 \mathrm{sec}$ and incubated in a $100 \mu \mathrm{l}$ volume of water overnight at $4^{\circ} \mathrm{C}$. A total of $5 \mu \mathrm{l}$ of each sample was reamplified with $50 \mathrm{ng}$ of the same primers used in the preamplification procedure, $1.5 \mathrm{mM} \mathrm{Mg}{ }^{2+}, 0.2 \mathrm{mM}$ dNTP mix and $1 \mathrm{U}$ of Taq polymerase in a $20 \mu \mathrm{l}$ total reaction volume. The PCR reaction was conducted using the following conditions: $94^{\circ} \mathrm{C}$ for $2 \mathrm{~min}$; 35 cycles at: $94^{\circ} \mathrm{C}$ for $30 \mathrm{sec}$, $56^{\circ} \mathrm{C}$ for $1 \mathrm{~min}$ and $72^{\circ} \mathrm{C}$ for $1 \mathrm{~min}$, followed by $72^{\circ} \mathrm{C}$ for $7 \mathrm{~min}$. A $5 \mu \mathrm{l}$ aliquot of the PCR mixture was run on an agarose gel, and the remaining reactions were purified with PolyEthylene Glycol (PEG) 8000. The cDNA fragments were eluted in $10 \mu \mathrm{l}$ of $\mathrm{H}_{2} \mathrm{O}$ and either directly sequenced or sequenced after subcloning into the pGEM-T Easy Vector (Promega) followed by plasmid purification with QIAprep Spin Miniprep kit (Qiagen). The fragments were sequenced using the automated sequencer 3130 Genetic Analyser (Applied Biosystems, Foster City, CA). 
The sequences were aligned to the non-redundant protein sequences (nr) database at the NCBI [77] using BLASTX, which also calculated protein similarity and predicted the hypothetical function of the unknown fragment sequences.

All sequences differentially expressed in cDNA-AFLP analysis were annotated according to the three main gene ontological categories (cellular component, biological process and molecular function) using the Blast2GO software v1.3.3 [78,79] according to Galla and coworkers [31], with minor modifications. All sequences were also aligned to the OLEA EST database using the BlastN algorithm to detect possible homologous transcripts.

Enzyme mapping of the annotated sequences was performed using a direct GO to Enzyme annotation, and this programme was also used to query the KEGG maps to define the main metabolic pathways involved. The conserved domains were identified using the Conserved Domain Database (CDD) [80,81].

Sequences showing expression profiles compatible with the content of corresponding metabolites were further characterised. Fragments were extended at 3' and 5' ends using a RACE-PCR method to obtain long partial or fulllength cDNA clones. The resulting sequences were used to confirm the putative function of each compound. RACEPCR was performed using the SMART RACE CDNA Amplification Kit (Clontech), with some modifications. Briefly, total RNA ( $2 \mu \mathrm{g}$ ) was reverse transcribed using the 3'CDS primer II A and the SMART II A oligonucleotide (Clontech). The reactions were incubated in a solution containing $50 \mathrm{mM}$ Tris- $\mathrm{HCl} \mathrm{pH} \mathrm{8.3,} 75 \mathrm{mM} \mathrm{KCl,} 6 \mathrm{mM}$ $\mathrm{MgCl}_{2}$ and $2 \mathrm{mM}$ DTT for $1.5 \mathrm{~h}$ at $42^{\circ} \mathrm{C}$ with Superscript III (Invitrogen). For the second strand synthesis, touchdown PCR was performed using a small aliquot (1/20 volume) of the primary template with EX Taq (Takara), the 5' PCR primer II A (Clontech) and a gene specific primer. A thermal cycling programme was performed using the following conditions: an initial denaturation step of $94^{\circ} \mathrm{C}$ for $2 \mathrm{~min}$, followed by 16 cycles of $94^{\circ} \mathrm{C}$ for $30 \mathrm{sec}, 68^{\circ} \mathrm{C}$ for $30 \mathrm{sec}$ with a $0.5^{\circ} \mathrm{C}$ decrease per cycle and $72^{\circ} \mathrm{C}$ for $3 \mathrm{~min}$. An additional 20 cycles were performed at $94^{\circ} \mathrm{C}$ for $30 \mathrm{sec}, 60^{\circ} \mathrm{C}$ for $30 \mathrm{sec}$ and $72^{\circ} \mathrm{C}$ for $3 \mathrm{~min}$, with a final elongation step at $72^{\circ} \mathrm{C}$ for $5 \mathrm{~min}$. Nested PCR was required in some cases to improve the specificity of the amplification. The samples were purified using PEG 8000 and either directly sequenced or sequenced after being cloned into the pGEM-T Easy vector with the 3130 Genetic Analyser (Applied Biosystems, Foster City, CA).

\section{Semiquantitative RT-PCR}

To validate the differential expression patterns of cDNAAFLP selected clones, preliminary semi-quantitative PCR experiments were performed on 24 transcripts using different biological replicates of the samples. The RNeasy Plant
Mini Kit (Qiagen) was used to isolate $2 \mu \mathrm{g}$ of total RNA from biological replicates of the olive samples used in the cDNA-AFLP analysis. The cDNA synthesis was performed using SuperScript III according to the manufacturer's protocol. Elongation Factor $(E F 1 \alpha)$ was used as the endogenous gene for sample normalisation [31]. Specific EF1 $\alpha$ primers were used for cDNA sample normalisation with the following conditions: a $25 \mu$ l total reaction volume containing $10 \mu \mathrm{l}$ of first strand cDNA diluted 1:50, $1.5 \mathrm{mM} \mathrm{Mg}^{2+}, 0.2 \mathrm{mM}$ dNTP mix and $1.25 \mathrm{U}$ of Taq polymerase (Invitrogen). The following PCR amplification conditions were used: $94^{\circ} \mathrm{C}$ for $3 \mathrm{~min}, 20$ to 45 cycles (depending on the amplicon signal intensity obtained under non-saturating PCR conditions) at $94^{\circ} \mathrm{C}$ for $30 \mathrm{sec}$, $60^{\circ} \mathrm{C}$ for $30 \mathrm{sec}$ and $72^{\circ} \mathrm{C}$ for $50 \mathrm{sec}$, followed by $72^{\circ} \mathrm{C}$ for $7 \mathrm{~min}$. The cDNAs were normalised based upon band intensity. After sample normalisation, a PCR was performed using the previously described conditions with 16-bp specific primers that included the restriction site sequences (EcoI and MseI) used for the cDNA-AFLP analysis and a fragment specific region (Additional file 13) to validate both the expression and the allelic polymorphisms of any genes of interest.

\section{Quantitative RT-qPCR}

The RT-qPCR experiments were performed on the most representative candidates of main branches of secondary metabolites maps. RT-qPCR was performed on the subset of most robust 37 putative transcripts to verify if their expression profiles corresponded to the patterns of secoiridoid, phenolics and other secondary metabolites synthesis or degradation. Coratina and Dolce d'Andria were used as reference HP and LP varieties, respectively. Total RNA was extracted from $0.2 \mathrm{~g}$ of fruit mesocarp and exocarp with the RNeasy Plant Mini Kit (Qiagen) and treated with DNase I (Qiagen). Reverse transcription of $2 \mu \mathrm{g}$ of RNA was performed using oligo $(\mathrm{dT})_{18}$ and the SuperScript III Reverse Transcriptase kit (Invitrogen) according to the manufacturer's instructions. Quantitative real-time PCR was performed on a PCR Real Time 7300 (Applied Biosystems, Foster City, CA) according to the manufacturer's protocol and using the Reagent kit for SYBR Green analysis (Applied Biosystems) and genespecific primers (Additional file 14). Primers were verified by the presence of a single PCR product band after agarose gel electrophoresis. All reactions were performed in triplicate. After each assay, a dissociation kinetics analysis was performed to verify the specificity of the amplification products. Relative amounts of all mRNAs were calculated using the $2^{-\Delta \Delta C T}$ method [82], where $\Delta \mathrm{Ct}=\mathrm{Ct}_{\text {target }}$ gene $-\mathrm{Ct}_{\text {reference gene }}$ using Dolce D'Andria at 165 DAF as a control sample. The housekeeping Elongation Factor $1-\alpha$ gene was used as an endogenous reference gene for cDNA normalisation. The 
data for three biological replicates were analysed using an analysis of variance (ANOVA) followed by Bonferroni's post hoc test $(\mathrm{P}<0.05)$ with $\mathrm{R}$ software (version 2.14.0) [83].

\section{Additional files}

Additional file 1: Chemical structures of main phenolic compounds of olive fruits. Secoiridoid glucosides (oleuropein, demethyloleuropein, ligstroside) and verbascoside (hydroxycinnamic derivative observed in olive fruits) (from Servili et al. [5]).

Additional file 2: Putative biochemical mechanism of secoiridoid derivatives formation. Figure from Servili et al. [5].

Additional file 3: Mean concentration of total phenolics in mesocarp and exocarp of olive fruits during fruit development.

Additional file 4: Pinoresinol and acetoxypinoresinol content in olive fruits. Pinoresinol (A) and acetoxypinoresinol (B) contents in the 12 cultivars during fruit ripening $(45,60,75,90,105,120,135,150$ and 165 DAF) were considered. Red and blue lines represent high (HP) and low phenolic (LP) cultivars, respectively. Standard errors are not shown in the graphs because these values were lower than $5 \%$.

\section{Additional file 5: Transcripts selected from OLEA EST database.}

Additional file 6: Transcript-derived fragments (TDFs) obtained by cDNA-AFLP analysis. List of the fragments showing similarity to known proteins, their putative functions, Genbank accession numbers, their expression trends and homologues in OLEA database.

Additional file 7: $\mathrm{GO}$ terms distribution. $\mathrm{GO}$ terms distribution in the biological processes (A), molecular functions (B) and cellular components (C) vocabularies.

Additional file 8: Comparison between CDNA-AFLP (A) and SQ-PCR (B) revealed the same expression pattern. Some examples are reported for LP (Dolce d'Andria and Tendellone) and HP (Coratina and Rosciola) cultivars at three stages of fruit ripening $(1,2,3$ correspond to 45, 90, 165 DAF, respectively). Elongation Factor 1a was used as a reference gene (C). The putative heat shock proteins purple acid phosphatase, 1,3- $\beta$-glucosidase, ferredoxin chloroplast precursor, polygalacturonase, and 4-hydroxy-3-methylbut-2-enyl diphosphate reductase are reported.

Additional file 9: Biosynthesis of isoprenic unit of secoiridoids. The MEP pathway for isoprenoid biosynthesis is reported. Through DXP synthesis and reduction, MEP is obtained and converted to CDPME through the transfer of a phosphocytidyl moiety. CDPME is phosphorylated to CDPMEP and cyclised to MECP. After an oxidoreduction reaction CDPMES is reduced to HMBPP, which is finally converted to IPP or DMAPP.

Additional file 10: Biosynthesis of terpenic moiety of secoiridoids. The biosynthetic steps for the production of the terpenic moiety of oleuropein in Olea europaea according to Obied et al. [1] is reported, with some modifications. Based on our data, we propose that in the olive fruits geraniol for the secoiridoid synthesis derives from the MEP pathway. Iridoidal is produced through a series of hydroxylation and oxidation reactions on geraniol followed by a cyclisation reaction. Further oxidation yields iridotrial and deoxyloganic acid aglycone. Deoxyloganic acid is converted to 7-epi-loganic acid through the hydroxylation of the cyclopentane ring, then the hydroxyl group is oxidised to form 7-ketologanic acid. An esterification reaction is required for the conversion of 7-ketologanic acid to 7-ketologanin, and subsequently, oleoside 11-methyl ester is produced through the oxidation of the ketonic group. In a reaction catalysed by glucosyl transferase, oleoside 11-methyl ester is converted to 7- $\beta$-1-D-glucopyranosyl 11-methyl oleoside, which is the precursor of ligstroside and oleuropein.

Additional file 11: Biosynthesis of phenolic moiety of secoiridoids. The biosynthetic pathway for the production of oleuropein and 3,4-DHPEA-EDA in Olea europaea, according to Ryan et al. [8], is reported. First $p$-hydroxyphenylalanine (tyrosine) is deaminated and oxidised by the enzyme amine oxidase to form $p$-hydroxyphenylpyruvic acid. Subsequently, $p$-hydroxyphenylacetic acid is generated through the decarboxylation of $p$-hydroxyphenylpyruvic acid. By reduction of $p$-hydroxyphenylacetic acid, $p$-hydroxyphenylethanol (tyrosol) is formed, and through a series of condensation reactions with oleoside, this product produces ligstroside, 3,4-DHPEA-EDA, and oleuropein.

Additional file 12: Primers used for the amplification of transcripts involved in secondary metabolite synthesis in olive. Primer sequences and amplicon size are provided.

Additional file 13: Primers used for RT-sqPCR analyses. Primer sequences and amplicon sizes are provided.

Additional file 14: Primers used for RT-qPCR analyses. Primer sequences and amplicon sizes are provided.

\section{Abbreviations}

DAF: Days after flowering; HP: High phenolics; LP: Low phenolics; cvs: Cultivars; RT-qPCR: Real-time reverse transcription polymerase chain reaction; G3P: Glyceraldehyde 3-phosphate; DXP: 1-deoxy-D-xylulose-5-P; DXS: 1-deoxy-D-xylulose-5-P synthase; DXR: 1-deoxy-D-xylulose-5-P reductoisomerase; MEP: 2-C-methyl-D-erythritol-4-P; CDPME: 4-(CDP)-2-Cmethyl-D-erythritol; CDPMES: 2-C-methyl-D-erythritol 4-phosphate cytidyltransferase; CDPMEK: 4-(CDP)-2-C-methyl-D-erythritol kinase; CDPME2P: 4-(CDP)-2-C-methyl-D-erythritol-2-P; MECP: 2-C-methyl-D-erythritol 2,4-cyclo-PP; MECPS: 2-C-methyl-D-erythritol 2,4-cyclodiphosphate synthase; HMBPP: 1-hydroxy-2-methyl-2-(E)-butenyl-4-PP; HMBPPS: 1-hydroxy-2-methyl2-(E)-butenyl-4-PP synthase; HMBPPR: 1-hydroxy-2-methyl-2-(E)-butenyl-4-PP reductase; DMAPP: Dimethylallyl diphosphate; IPP: Isopentenyl diphosphate; IPPI: Isopentenyl diphosphate delta isomerase; AC: Acetyl-CoA; ACC: Acetoacetyl-CoA; HMG: 3-hydroxy-3-methylglutaryl-CoA; HMGR: HMGC reductase; MVAK: MVA kinase; MVAP: Mevalonate phosphate; MVAPK: MVAP kinase; MVAPP: Mevalonate diphosphate; MVAPPD: Mevalonate diphosphate decarboxylase; DAPP: Dimethylallyl diphosphate; GES: Geraniol synthase; G10H: Geraniol 10-hydroxylase; NDH1: NADH dehydrogenase I; GT: Glucosyltransferase; SLS: Secologanin synthase; LAMT: Loganic acid methyltransferase; ADH: Arogenate dehydrogenase; CuAO: Copper amine oxidase; $p$-HPPA: $p$-hydroxyphenylpyruvic acid; $p$-HPAA: $p$ -

hydroxyphenylacetic acid; TYRD: Tyrosine/dopa decarboxylase; ALDH: Alcohol dehydrogenase; PPO: Polyphenol oxidase; PAL: Phenylalanine ammonia-lyase; 4CL: 4-coumarate coenzyme A ligase; GPP: Geranyl diphosphate; LS: Limonene synthase; FPP: Farnesyl diphosphate; FPPS: Farnesyl diphosphate synthase; GGPP: Geranyl geranyl pyrophosphate; GGPS: Geranyl geranyl pyrophosphate synthase; SQS: Squalene synthase; LUPS: Lupeol synthase.

\section{Competing interests}

The authors declare that they have no competing interests.

\section{Authors' contributions}

LB, MS, FA conceived the research plan. SC, SU, GV, SE, AT performed the analyses of olive phenolic compounds by HPLC. AR and SC monitored and controlled tree water status of plants. FA performed CDNA-AFLP analyses. FA and FP performed the identification of candidate genes from EST data. FA and RM performed the RT-qPCR experiments and the re-sequencing of candidate genes. FP and FA performed the statistical analyses on RT-qPCR data. FA and LB drafted the manuscript. FP, GP, MS, AR and RR critically revised the manuscript. All authors read and approved the manuscript.

\section{Acknowledgments}

The research was supported by the Project OLEA - Genomics and Breeding of Olive, funded by MIPAF, Italy and by the Project FISR - Improving flavour and nutritional properties of plant food after first and second transformation, Italian Ministry of Research.

\section{Author details}

${ }^{1}$ CNR - Institute of Plant Genetics, 06128 Perugia, Italy. ${ }^{2}$ Dept. of Economical and Food Science, University of Perugia, 06126 Perugia, Italy. ${ }^{3}$ CRA -OLI, 06049 Spoleto, PG, Italy. ${ }^{4}$ Dept. of Soil, Plant, Environment and Animal Production Sciences, University of Naples 'Federico II', 80055 Portici, NA, Italy. ${ }^{5}$ ENEA, TRISAIA Research Center, 75026 Rotondella, Matera, Italy. 
Received: 26 April 2012 Accepted: 30 August 2012

Published: 10 September 2012

\section{References}

1. Obied HK, Prenzler PD, Ryan D, Servili M, Taticchi A, Esposto S, Robards K: Biosynthesis and biotransformations of phenol-conjugated oleosidic secoiridoids from Olea europaea L. Nat Prod Rep 2008, 25(6):1167-1179.

2. Beauchamp GK, Keast RS, Morel D, Lin J, Pika J, Han Q, Lee CH, Smith AB, Breslin PA: Phytochemistry: ibuprofen-like activity in extra-virgin olive oil. Nature 2005, 437(7055):45-46.

3. Oliveras López MJ, Innocenti M, leri F, Giaccherini C, Romani A, Mulinacci N: HPLC/DAD/ESI/MS detection of lignans from Spanish and Italian Olea europaea L. fruits. Journal of Food Composition and Analysis 2008, 21:62-70.

4. Owen RW, Haubner R, Mier W, Giacosa A, Hull WE, Spiegelhalder B, Bartsch $\mathrm{H}$ : Isolation, structure elucidation and antioxidant potential of the major phenolic and flavonoid compounds in brined olive drupes. Food Chem Toxicol 2003, 41(5):703-717.

5. Servili M, Selvaggini R, Esposto S, Taticchi A, Montedoro G, Morozzi G: Health and sensory properties of virgin olive oil hydrophilic phenols: agronomic and technological aspects of production that affect their occurrence in the oil. J Chromatogr A 2004, 1054(1-2):113-127.

6. Boskou G, Salta FN, Chrysostomou S, Mylona A, Chiou A, Andrikopoulos NK: Antioxidant capacity and phenolic profile of table olives from the Greek market. Food Chem 2006, 94:558-564

7. Jerman T, Trebše P, Mozetic ${ }^{2}$ Vodopivec B: Ultrasound-assisted solid liquid extraction (USLE) of olive fruit (Olea europaea) phenolic compounds. Food Chem 2010, 123(123):175-182.

8. Ryan D, Antolovich M, Herlt T, Prenzler PD, Lavee S, Robards K Identification of phenolic compounds in tissues of the novel olive cultivar hardy's mammoth. J Agric Food Chem 2002, 50(23):6716-6724.

9. Servili M, Baldioli M, Selvaggini R, Macchioni A, Montedoro G: Phenolic compounds of olive fruit: one- and two-dimensional nuclear magnetic resonance characterization of Nuzhenide and its distribution in the constitutive parts of fruit. J Agric Food Chem 1999, 47(1):12-18.

10. Goulas $\vee$, Manganaris GA: Towards an Efficient Protocol for the Determination of Triterpenic Acids in Olive Fruit: A Comparative Study of Drying and Extraction Methods. Phytochem Anal 2012, 23(5):444-449.

11. Dabbou S, Issaoui M, Esposto S, Sifi S, Taticchi A, Servili M, Montedoro GF, Hammami M: Cultivar and growing area effects on minor compounds of olive oil from autochthonous and European introduced cultivars in Tunisia. J Sci Food Agric 2009, 89:1314-1325

12. Waterman E, Lockwood B: Active Components and Clinical Applications of Olive Oil. Altern Med Rev 2007, 12(4):331-342.

13. Stiti N, Triki S, Hartmann MA: Formation of triterpenoids throughout Olea europaea fruit ontogeny. Lipids 2007, 42(1):55-67.

14. Servili M, Montedoro GF: Contribution of phenolic compounds to virgin olive oil quality. European Journal of Lipid Science and Technology 2002, 104:602-613.

15. Omar SH: Oleuropein in olive and its pharmacological effects. Sci Pharm 2010, 78(2):133-154

16. Puel C, Mathey J, Agalias A, Kati-Coulibaly S, Mardon J, Obled C, Davicco MJ, Lebecque P, Horcajada MN, Skaltsounis AL, et al: Dose-response study of effect of oleuropein, an olive oil polyphenol, in an ovariectomy/ inflammation experimental model of bone loss in the rat. Clin Nutr 2006 25(5):859-868

17. Mumm R, Hilker M: Direct and indirect chemical defence of pine against folivorous insects. Trends Plant Sci 2006, 11(7):351-358.

18. Umehara M, Hanada A, Yoshida S, Akiyama K, Arite T, Takeda-Kamiya N, Magome H, Kamiya Y, Shirasu K, Yoneyama K, et al: Inhibition of shoot branching by new terpenoid plant hormones. Nature 2008, 455(7210):195-200

19. Beckman $\mathrm{CH}$ : Phenolic-storing cells: keys to programmed cell death and periderm formation in wilt disease resistance and in general defence responses in plants? Physiological and Molecular Plant Pathology 2000 57:101-110.

20. Franceschi VR, Krokene P, Christiansen E, Krekling T: Anatomical and chemical defenses of conifer bark against bark beetles and other pests. New Phytol 2005, 167(2):353-375.

21. Ockels FS, Eyles A, McPherson BA, Wood DL, Bonello P: Phenolic chemistry of coast live oak response to Phytophthora ramorum infection. $J$ Chem Ecol 2007, 33(9):1721-1732
22. Wallis C, Eyles A, Chorbadjian R, McSpadden Gardener B, Hansen R, Cipollini D, Herms DA, Bonello P: Systemic induction of phloem secondary metabolism and its relationship to resistance to a canker pathogen in Austrian pine. New Phytol 2008, 177(3):767-778.

23. Kubo I, Matsumoto A, Takase I: A multichemical defense mechanism of bitter olive Olea europaea (Oleaceae). Is oleuropein a phytoalexin precursor? J Chem Ecol 1985, 11:251-263.

24. Konno K, Hirayama C, Yasui H, Nakamura M: Enzymatic activation of oleuropein: a protein crosslinker used as a chemical defense in the privet tree. Proc Natl Acad Sci 1999, 96(16):9159-9164.

25. Damtoft S, Franzyk H, Jensen SR: Biosynthesis of secoiridoids in Fontanesia. Phytochemistry 1995, 38(3):615-621.

26. Damtoft S, Franzyk H, Jensen SR: Biosynthesis of iridoids in Syringa and Fraxinus: carbocyclic iridoid precursors. Phytochemistry 1995, 40(3):785-792.

27. Malik NSA, Bradford JM: Recovery and stability of oleuropein and other phenolic compounds during extraction and processing of olive (Olea europaea L.) leaves. Journal of Food Agriculture and Environment 2008, 6:8-13.

28. Shibuya M, Zhang H, Endo A, Shishikura K, Kushiro T, Ebizuka Y: Two branches of the lupeol synthase gene in the molecular evolution of plant oxidosqualene cyclases. Eur J Biochem 1999, 266(1):302-307.

29. Saimaru H, Orihara Y, Tansakul P, Kang YH, Shibuya M, Ebizuka Y: Production of triterpene acids by cell suspension cultures of Olea europaea. Chem Pharm Bull(Tokyo) 2007, 55(5):784-788.

30. Alagna F, D'Agostino N, Torchia L, Servili M, Rao R, Pietrella M, Giuliano G, Chiusano ML, Baldoni L, Perrotta G: Comparative 454 pyrosequencing of transcripts from two olive genotypes during fruit development. BMC Genomics 2009, 10:399.

31. Galla G, Barcaccia G, Ramina A, Collani S, Alagna F, Baldoni L, Cultrera NG, Martinelli $F$, Sebastiani $L$, Tonutti P: Computational annotation of genes differentially expressed along olive fruit development. BMC Plant Biol 2009, 9:128.

32. Saito K, Hirai MY, Yonekura-Sakakibara K: Decoding genes with coexpression networks and metabolomics - 'majority report by precogs'. Trends Plant Sci 2008, 13(1):36-43

33. Morello JR, Romero MP, Motilva MJ: Effect of the maturation process of the olive fruit on the phenolic fraction of drupes and oils from Arbequina, Farga, and Morrut cultivars. J Agric Food Chem 2004, 52(19):6002-6009.

34. Jemai H, Bouaziz M, Sayadi S: Phenolic composition, sugar contents and antioxidant activity of Tunisian sweet olive cultivar with regard to fruit ripening. J Agric Food Chem 2009, 57(7):2961-2968.

35. The OLEA EST database: The OLEA EST database. http://140.164.45.140/oleaestdb/.

36. Collu G, Unver N, Peltenburg-Looman AM, van der Heijden R, Verpoorte R, Memelink J: Geraniol 10-hydroxylase, a cytochrome P450 enzyme involved in terpenoid indole alkaloid biosynthesis. FEBS Lett 2001, 508(2):215-220.

37. Irmler S, Schroder G, St-Pierre B, Crouch NP, Hotze M, Schmidt J, Strack D, Matern U, Schroder J: Indole alkaloid biosynthesis in Catharanthus roseus: new enzyme activities and identification of cytochrome P450 CYP72A1 as secologanin synthase. Plant J 2000, 24(6):797-804

38. Murata J, Roepke J, Gordon H, De Luca V: The leaf epidermome of Catharanthus roseus reveals its biochemical specialization. Plant Cell 2008, 20(3):524-542.

39. Walter $\mathrm{MH}$, Hans J, Strack D: Two distantly related genes encoding 1-deoxy-d-xylulose 5-phosphate synthases: differential regulation in shoots and apocarotenoid-accumulating mycorrhizal roots. Plant J 2002, 31(3):243-254

40. Cordoba E, Salmi M, Leon P: Unravelling the regulatory mechanisms that modulate the MEP pathway in higher plants. J Exp Bot 2009, 60(10):2933-2943.

41. Estevez JM, Cantero A, Reindl A, Reichler S, Leon P: 1-Deoxy-D-xylulose-5phosphate synthase, a limiting enzyme for plastidic isoprenoid biosynthesis in plants. J Biol Chem 2001, 276(25):22901-22909.

42. Xing S, Miao J, Li S, Qin G, Tang S, Li H, Gu H, Qu L: Disruption of the 1deoxy-D-xylulose-5-phosphate reductoisomerase (DXR) gene results in albino, dwarf and defects in trichome initiation and stomata closure in Arabidopsis. Cell Res 2010, 20(6):688-700. 
43. Zulak KG, Bohlmann J: Terpenoid biosynthesis and specialized vascular cells of conifer defense. J Integr Plant Biol 2010, 52(1):86-97.

44. Battilana J, Costantini L, Emanuelli F, Sevini F, Segala C, Moser S, Velasco R, Versini G, Stella Grando M: The 1-deoxy-D: -xylulose 5-phosphate synthase gene co-localizes with a major QTL affecting monoterpene content in grapevine. Theor Appl Genet 2009, 118(4):653-669.

45. Emanuelli F, Battilana J, Costantini L, Le Cunff L, Boursiquot JM, This P, Grando MS: A candidate gene association study on muscat flavor in grapevine (Vitis vinifera L.). BMC Plant Biol 2010, 10:241.

46. Tiski I, Marraccini P, Pot D, Vieira LG, Pereira LF: Characterization and expression of two CDNA encoding 3-Hydroxy-3-methylglutaryl coenzyme A reductase isoforms in coffee (Coffea arabica L.). Omics 2011, 15(10):719-727.

47. Aharoni A, Jongsma MA, Kim TY, Ri MB, Giri AP, Verstappen FWA, Schwab W, Bouwmeester HJ: Metabolic engineering of terpenoid biosynthesis in plants. Phytochemistry Reviews 2006, 5:49-58

48. Dudareva N, Andersson S, Orlova I, Gatto N, Reichelt M, Rhodes D, Boland W, Gershenzon J: The nonmevalonate pathway supports both monoterpene and sesquiterpene formation in snapdragon flowers. Proc Natl Acad Sci U S A 2005, 102(3):933-938.

49. Damtoft $\mathrm{S}$, Franzyk $\mathrm{H}$, Jensen SR: Biosynthesis of secoiridoid glucosides in Oleaceae. Phytochemistry 1993, 34(5):1291-1299.

50. lijima Y, Gang DR, Fridman E, Lewinsohn E, Pichersky E: Characterization of geraniol synthase from the peltate glands of sweet basil. Plant Physiol 2004, 134(1):370-379.

51. Sung PH, Huang FC, Do YY, Huang PL: Functional expression of geraniol 10-hydroxylase reveals its dual function in the biosynthesis of terpenoid and phenylpropanoid. J Agric Food Chem 2011, 59(9):4637-4643.

52. Jensen SR, Franzyk H, Wallander E: Chemotaxonomy of the Oleaceae: iridoids as taxonomic markers. Phytochemistry 2002, 60(3):213-231

53. Soler-Rivas C, Espìn JC, Wichers HJ: Oleuropein and related compounds. $J$ Sci Food Agric 2000, 80:1013-1023.

54. Vezzaro A, Krause ST, Nonis A, Ramina A, Degenhardt J, Ruperti B: Isolation and characterization of terpene synthases potentially involved in flavor development of ripening olive (Olea europaea) fruits. J Plant Physiol 2012, 169(9):908-914.

55. Ryan D, Prenzler PD, Lavee S, Antolovich M, Robards K: Quantitative changes in phenolic content during physiological development of the olive (Olea europaea) cultivar Hardy's Mammoth. J Agric Food Chem 2003, 51(9):2532-2538

56. O'Connor SE, Maresh JJ: Chemistry and biology of monoterpene indole alkaloid biosynthesis. Nat Prod Rep 2006, 23(4):532-547.

57. Legrand P, Dumas R, Seux M, Rippert P, Ravelli R, Ferrer JL, Matringe M: Biochemical characterization and crystal structure of Synechocystis arogenate dehydrogenase provide insights into catalytic reaction. Structure 2006, 14(4):767-776

58. Rippert P, Puyaubert J, Grisollet D, Derrier L, Matringe M: Tyrosine and phenylalanine are synthesized within the plastids in Arabidopsis. Plant Physiol 2009, 149(3):1251-1260.

59. Saimaru H, Orihara Y: Biosynthesis of acteoside in cultured cells of Olea europaea. J Nat Med 2010, 64(2):139-145.

60. Zhang JX, Ma LQ, Yu HS, Zhang H, Wang HT, Qin YF, Shi GL, Wang YN: A tyrosine decarboxylase catalyzes the initial reaction of the salidroside biosynthesis pathway in Rhodiola sachalinensis. Plant Cell Rep 2011, 30(8):1443-1453.

61. Rippert $\mathrm{P}$, Matringe $\mathrm{M}$ : Molecular and biochemical characterization of an Arabidopsis thaliana arogenate dehydrogenase with two highly similar and active protein domains. Plant Mol Biol 2002, 48(4):361-368

62. Sebela M, Frebort I, Lemr K, Brauner F, Pec P: A study on the reactions of plant copper amine oxidase with $\mathrm{C} 3$ and $\mathrm{C} 4$ aliphatic diamines. Arch Biochem Biophys 2000, 384(1):88-99.

63. Ortega-Garcia F, Blanco S, Peinado MA, Peragon J: Polyphenol oxidase and its relationship with oleuropein concentration in fruits and leaves of olive (Olea europaea) cv. 'Picual' trees during fruit ripening. Tree Physiol 2008, 28(1):45-54.

64. Guillet G, Poupart J, Basurco J, De Luca V: Expression of tryptophan decarboxylase and tyrosine decarboxylase genes in tobacco results in altered biochemical and physiological phenotypes. Plant Physiol 2000, 122(3):933-943.
65. Facchini PJ, De Luca V: Differential and tissue-specific expression of a gene family for tyrosine/dopa decarboxylase in opium poppy. J Biol Chem 1994, 269(43):26684-26690.

66. Agati G, Pinelli P, Cortes Ebner S, Romani A, Cartelat A, Cerovic ZG Nondestructive evaluation of anthocyanins in olive (Olea europaea) fruits by in situ chlorophyll fluorescence spectroscopy. J Agric Food Chem 2005, 53(5)):1354-1363.

67. Gomez-Rico A, Salvador MD, Fregapane G: Effect of cultivar and ripening on minor components in Spanish olive fruits and their corresponding virgin olive oils. Food Res Int 2008, 41:433-440.

68. Kumar A, Ellis BE: 4-coumarate:CoA ligase gene family in Rubus idaeus: cDNA structures, evolution, and expression. Plant Mol Biol 2003, 51(3):327-340

69. Sivakumar G, Uccella N, Briccoli Bati C: Demethyloleuropein and betaglucosidase activity in olive fruits. Biotechnol J 2007, 2(3):381-385.

70. Flamini G, Cioni PL, Morelli I: Volatiles from leaves, fruits, and virgin oil from Olea europaea Cv. Olivastra Seggianese from Italy. J Agric Food Chem 2003, 51(5)):1382-1386.

71. Roca M, Minguez-Mosquera Ml: Carotenoid levels during the period of growth and ripening in fruits of different olive varieties (Hojiblanca, Picual and Arbequina). J Plant Physiol 2003, 160:451-459.

72. Servili M, Badioli M, Selvaggini R, Miniati E, Macchioni A, Montedoro GF: High-Performance Liquid Chromatography evaluation of phenols in olive fruits, virgin olive oil, vegetation waters, and pomace and ID- and 2DNuclear Magnetic Resonance characterization. J Am Oil Chem Soc 1999, 7(76):873-882.

73. Selvaggini R, Servili M, Urbani S, Esposto S, Taticchi A, Montedoro G: Evaluation of phenolic compounds in virgin olive oil by direct injection in high-performance liquid chromatography with fluorometric detection. J Agric Food Chem 2006, 54(8):2832-2838.

74. The Kyoto Encyclopaedia of Genes and Genomes (KEGG). http://www.genome.jp/kegg.

75. Kanehisa M, Araki M, Goto S, Hattori M, Hirakawa M, Itoh M, Katayama T, Kawashima S, Okuda S, Tokimatsu T, et al: KEGG for linking genomes to life and the environment. Nucleic Acids Res 2008, 36(Database issue):D480-D484.

76. Bachem CW, van der Hoeven RS, de Bruijn SM, Vreugdenhil D, Zabeau M, Visser RG: Visualization of differential gene expression using a novel method of RNA fingerprinting based on AFLP: analysis of gene expression during potato tuber development. Plant J 1996, 9(5):745-753.

77. The National Center for Biotechnological Information (NCBI). http://blast.ncbi.nlm.nih.gov.

78. Conesa A, Gotz S, Garcia-Gomez JM, Terol J, Talon M, Robles M: Blast2GO: a universal tool for annotation, visualization and analysis in functional genomics research. Bioinformatics 2005, 21(18):3674-3676.

79. The Blast2GO software. http://www.blast2go.org].

80. The Conserved Domain Database (CDD): The Conserved Domain Database (CDD). http://www.ncbi.n/m.nih.gov/Structure/cdd/wrpsb.cgi].

81. Marchler-Bauer A, Anderson JB, Chitsaz F, Derbyshire MK, DeWeese-Scott C, Fong JH, Geer LY, Geer RC, Gonzales NR, Gwadz M, et al: CDD: specific functional annotation with the Conserved Domain Database. Nucleic Acids Res 2009, 37(Database issue)):D205-D210.

82. Livak KJ, Schmittgen TD: Analysis of relative gene expression data using real-time quantitative PCR and the 2(-Delta Delta $C(T)$ ) Method. Methods 2001, 25(4):402-408.

83. The R project. - www.r-project.org

\section{doi:10.1186/1471-2229-12-162}

Cite this article as: Alagna et al:: Olive phenolic compounds: metabolic and transcriptional profiling during fruit development. BMC Plant Biology 2012 12:162. 